MATHEMATICS OF COMPUTATION

Volume 77, Number 262, April 2008, Pages 611-632

S 0025-5718(07)02028-5

Article electronically published on October 18, 2007

\title{
A POSTERIORI ERROR ANALYSIS FOR CONFORMING MITC ELEMENTS FOR REISSNER-MINDLIN PLATES
}

\author{
C. CARSTENSEN AND JUN HU
}

\begin{abstract}
This paper establishes a unified a posteriori error estimator for a large class of conforming finite element methods for the Reissner-Mindlin plate problem. The analysis is based on some assumption (H) on the consistency of the reduction integration to avoid shear locking. The reliable and efficient a posteriori error estimator is robust in the sense that the reliability and efficiency constants are independent of the plate thickness $t$. The presented analysis applies to all conforming MITC elements and all conforming finite element methods without reduced integration from the literature.
\end{abstract}

\section{INTRODUCTION}

This paper is devoted to the finite element approximation for the ReissnerMindlin plate problem: Given $g \in L^{2}(\Omega)$ find $(\omega, \boldsymbol{\phi}) \in W \times \boldsymbol{\Theta}:=H_{0}^{1}(\Omega) \times H_{0}^{1}(\Omega)^{2}$ with

$$
a(\boldsymbol{\phi}, \boldsymbol{\psi})+(\boldsymbol{\gamma}, \nabla v-\boldsymbol{\psi})_{L^{2}(\Omega)}=(g, v)_{L^{2}(\Omega)} \quad \text { for all }(v, \boldsymbol{\psi}) \in W \times \boldsymbol{\Theta},
$$

and the shear force

$$
\gamma=\lambda t^{-2}(\nabla \omega-\phi)
$$

The bilinear form $a(\cdot, \cdot)$ models the linear elastic energy while $(\cdot, \cdot)_{L^{2}(\Omega)}$ denotes the $L^{2}$ scalar product. Here and throughout this paper, $\boldsymbol{\Theta}_{h} \subset \boldsymbol{\Theta}$ and $W_{h} \subset W$ denote some finite element spaces over some regular partition $\mathcal{T}_{h}$ while $\boldsymbol{R}_{h}$ denotes the reduction integration operator in the context of shear locking with values in the discrete shear force space $\boldsymbol{\Gamma}_{h}$. Some lower order examples of finite element spaces $W_{h}, \boldsymbol{\Theta}_{h}$, and the operator $\boldsymbol{R}_{h}: \boldsymbol{\Theta}_{h} \rightarrow \boldsymbol{\Gamma}_{h}$ are depicted in Table 1. We refer to Section 5 for further descriptions and for other discrete schemes.

The discrete problem reads: Find $\left(\omega_{h}, \phi_{h}\right) \in W_{h} \times \Theta_{h}$ with

$$
a\left(\phi_{h}, \boldsymbol{\psi}\right)+\left(\gamma_{h}, \nabla v-\boldsymbol{R}_{h} \boldsymbol{\psi}\right)=(g, v) \quad \text { for all }(v, \boldsymbol{\psi}) \in W_{h} \times \boldsymbol{\Theta}_{h}
$$

Received by the editor March 3, 2006 and, in revised form, November 11, 2006.

2000 Mathematics Subject Classification. Primary 65N30, 65N15, 35J25.

Key words and phrases. A posteriori, error analysis, Reissner-Mindlin Plate, MITC element.

The first author was supported by DFG Research Center MATHEON "Mathematics for key technologies" in Berlin.

The second author was partially supported by Natural Science Foundation of China under Grant 10601003 A Foundation for the Author of Excellent Doctoral Dissertation of PR China 200718. 
TABLE 1. Lower-order examples of finite element spaces $W_{h}, \boldsymbol{\Theta}_{h}$ and the reduction integration operator $\boldsymbol{R}_{h}: \boldsymbol{\Theta}_{h} \rightarrow \boldsymbol{\Gamma}_{h}$ in (1.3) and (1.4) with (H), namely $\boldsymbol{R}_{h} \boldsymbol{\Theta}_{h} \subset H_{0}(\operatorname{rot}, \Omega)$.

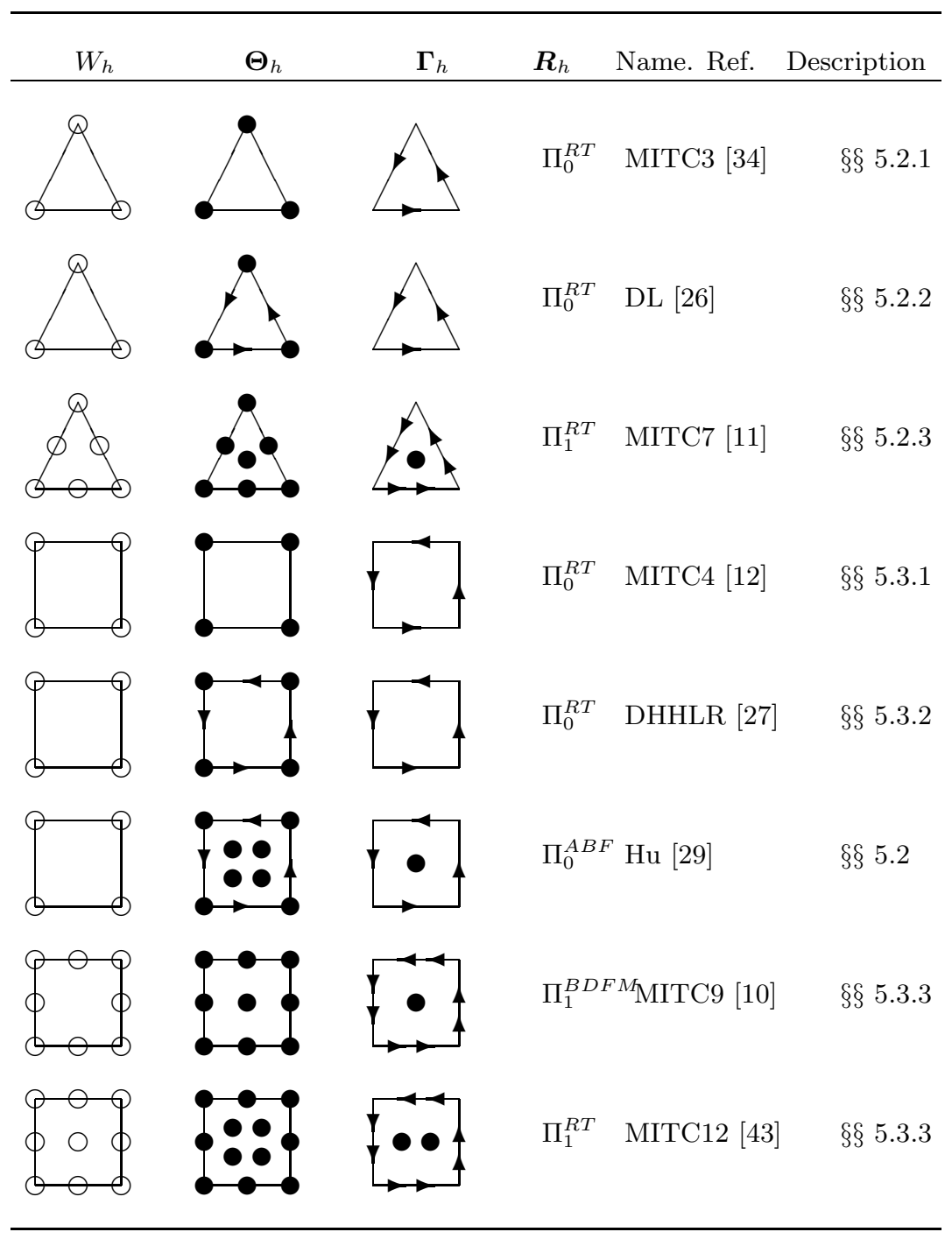

and the discrete shear force

$$
\gamma_{h}=\lambda t^{-2}\left(\nabla \omega_{h}-\boldsymbol{R}_{h} \phi_{h}\right) .
$$

This paper aims at a unified reliable and efficient residual-based a posteriori error analysis for a class of conforming elements for the Reissner-Mindlin problem under the hypothesis $(\mathrm{H})$ : For any $\boldsymbol{\psi}_{h} \in \boldsymbol{\Theta}_{h}$, it holds that

$$
\boldsymbol{R}_{h} \boldsymbol{\psi}_{h} \in H_{0}(\operatorname{rot}, \Omega),
$$

with the Sobolev space $H_{0}(\operatorname{rot}, \Omega)$ defined in Section 2.1. In fact, $(\underline{H})$ is satisfied for all the examples in Table 1 with detailed proofs given in Section 5 below. 
With the notation specified below, the main result of this paper implies that the error

$$
\begin{gathered}
\left\|\phi-\phi_{h}\right\|_{H^{1}(\Omega)}^{2}+\left\|\omega-\omega_{h}\right\|_{H^{1}(\Omega)}^{2}+t^{2}\left\|\boldsymbol{\gamma}-\gamma_{h}\right\|_{L^{2}(\Omega)}^{2} \\
+t^{4}\left\|\operatorname{rot}\left(\boldsymbol{\gamma}-\gamma_{h}\right)\right\|_{L^{2}(\Omega)}^{2}+\left\|\boldsymbol{\gamma}-\gamma_{h}\right\|_{H^{-1}(\Omega)}^{2}
\end{gathered}
$$

can be controlled by the error estimator

$$
\begin{aligned}
\eta_{h}^{2}= & : \sum_{E \in \mathcal{E}(\Omega)} h_{E}\left(\left\|\left[\mathcal{C} \varepsilon\left(\phi_{h}\right)\right] \cdot \nu_{E}\right\|_{L^{2}(E)}^{2}+\left(t^{2}+h_{E}^{2}\right)\left\|\left[\gamma_{h}\right] \cdot \nu_{E}\right\|_{L^{2}(E)}^{2}\right) \\
& +\sum_{K \in \mathcal{T}_{h}} h_{K}^{2}\left(\left\|\operatorname{div} \mathcal{C} \varepsilon\left(\phi_{h}\right)+\gamma_{h}\right\|_{L^{2}(K)}^{2}+\left(t^{2}+h_{K}^{2}\right)\left\|g+\operatorname{div} \gamma_{h}\right\|_{L^{2}(K)}^{2}\right) \\
& +\left\|\phi_{h}-\boldsymbol{R}_{h} \phi_{h}\right\|_{H(\mathrm{rot}, \Omega)}^{2}+\mu_{h}\left(\gamma_{h}\right)^{2}
\end{aligned}
$$

up to the data oscillation $\operatorname{osc}(g)$. Here and throughout this paper,

$$
\mu_{h}\left(\gamma_{h}\right):=\sup _{\psi_{h} \in\left(S_{0}^{1}\left(\mathcal{T}_{h}\right)\right)^{2} \backslash\{0\}} \frac{\left(\gamma_{h},\left(I-\boldsymbol{R}_{h}\right) \boldsymbol{\psi}_{h}\right)_{L^{2}(\Omega)}}{\left\|\boldsymbol{\psi}_{h}\right\|_{H^{1}(\Omega)}} .
$$

The operator $\mathcal{C}$ is defined in Subsection 2.1, and the lowest order conforming finite element space $S_{0}^{1}\left(\mathcal{T}_{h}\right)$ is defined in Subsection 2.2.

The estimator $\eta_{h}$ is robust in the sense that the reliability and efficiency constants are independent of the plate thickness $t$.

Remark 1.1. A different a posteriori error estimate was proposed for the DL element of 26 in 36. Instead of the norm with the term $\left\|\boldsymbol{\gamma}-\boldsymbol{\gamma}_{h}\right\|_{H^{-1}(\Omega)}$ here, the term $\| \boldsymbol{\gamma}-$ $\gamma_{h} \|_{H^{-1}(\operatorname{div}, \Omega)}$ was analyzed in [36]. It is unsatisfactory that no a priori convergence is known for $\left\|\gamma-\gamma_{h}\right\|_{H^{-1}(\mathrm{div}, \Omega)}$ for conforming MITC methods. This drawback motivated this paper with the focus on $\left\|\gamma-\gamma_{h}\right\|_{H^{-1}(\Omega)}$. The convergence in this norm is shown, for instance, in [18, Corollary 3.1] and [29, 30] for all examples (except MITC3) of this paper.

Remark 1.2. Compared to the analysis in [36, the analysis in this paper is based on three key arguments: an error representation formula, some mesh dependent norm, and some refined approximation of the Clément-type interpolation operator.

Remark 1.3. The last two terms of $\eta_{h}$ concern the consistency error from the reduction integration; they vanish for $\boldsymbol{R}_{h}=I$. Hence Theorems 2.1 and 2.2 below apply to any conforming finite element methods without reduction integration.

Remark 1.4. As we shall see in examples of Section [5, $\mu_{h}\left(\gamma_{h}\right)=0$ for high-order (triangle or quadrilateral) schemes. For the lower-order schemes, this term can be bounded by

$$
\begin{aligned}
\mu_{h}\left(\gamma_{h}\right)^{2} \lesssim & \sum_{E \in \mathcal{E}(\Omega)} h_{E}\left(t^{2}+h_{E}^{2}\right)\left\|\left[\gamma_{h}\right] \cdot \nu_{E}\right\|_{L^{2}(E)}^{2} \\
& +\sum_{K \in \mathcal{T}_{h}} h_{K}^{2}\left(t^{2}+h_{K}^{2}\right)\left\|g+\operatorname{div} \gamma_{h}\right\|_{L^{2}(K)}^{2} \quad+\text { h.o.t. }
\end{aligned}
$$

up to computable high-order terms h.o.t.

Here and throughout, an inequality $a \lesssim b$ replaces $a \leq C b$ with some multiplicative mesh-size independent constant $C>0$ that depends only on the domain $\Omega$ and the shape (e.g., through the aspect ratio) of elements $(C>0$ is also independent 
of crucial parameters as the plate thickness $t$ below). Finally, $a \approx b$ abbreviates $a \lesssim b \lesssim a$.

Remark 1.5. For the MITC methods under consideration, one has the following decomposition [18, 29, 30]: There exist unique $r \in H_{0}^{1}(\Omega), \boldsymbol{\alpha} \in \boldsymbol{H}_{0}(\operatorname{rot}, \Omega), r_{h} \in$ $W_{h}$, and $\boldsymbol{\alpha}_{h} \in \boldsymbol{\Gamma}_{h}$ with

$$
\begin{aligned}
& \boldsymbol{\gamma}=\nabla r+\boldsymbol{\alpha} \text { and }(\nabla r, \boldsymbol{\alpha})_{L^{2}(\Omega)}=0, \\
& \boldsymbol{\gamma}_{h}=\nabla r_{h}+\boldsymbol{\alpha}_{h} \text { and }\left(\nabla r_{h}, \boldsymbol{\alpha}_{h}\right)_{L^{2}(\Omega)}=0 .
\end{aligned}
$$

Therefore, the a priori convergence of $t^{2}\left\|\operatorname{rot}\left(\boldsymbol{\gamma}-\gamma_{h}\right)\right\|_{L^{2}(\Omega)}$ is assured by the convergence of $t^{2}\left\|\operatorname{rot}\left(\boldsymbol{\alpha}-\boldsymbol{\alpha}_{h}\right)\right\|_{L^{2}(\Omega)}$ proved, e.g, in [18, Theorem 3.2] for the MITC7, MITC9 and MITC12 element; cf. [29, 30] for the remaining elements (except MITC3).

Remark 1.6. Although the MITC3 element in Table 1 is unstable in the sense that there is no uniform a priori convergence with respect to the plate thickness $t$, the estimator $\eta_{h}$ is still reliable and efficient. At first glance this may be surprising, but it is a consequence that an a posteriori error analysis depends on the continuous operators and not on the stability of some discrete scheme.

Other a posteriori error estimators for the finite element methods of the ReissnerMindlin plate problem can be found in 21, 22, 37]. The paper [21] concerns the nonconforming Arnold-Falk element [4, and the paper [22] works on the stabilized method initiated in [3, 23]. Due to the lack of the convergence of $\left\|\gamma-\gamma_{h}\right\|_{H^{-1}(\text { div) }}$, both frameworks of [22] and 21] are inapplicable herein for the MITC methods. In 37, the authors discuss the a posteriori error estimator for the schemes based on the linked interpolation technique. The result is derived therein under some unproven saturation assumption which our work abandons . In addition, the norm analyzed in that paper contains the term $\sum_{K \in \mathcal{T}_{h}}\left(h_{K}+t\right)^{-2}\left\|\nabla\left(\omega-\omega_{h}\right)-\left(\phi-\phi_{h}\right)\right\|_{L^{2}(K)}^{2}$. The convergence of which is open for the MITC methods without stabilization.

The remaining part of the paper is organized as follows. We first introduce some notations and present the main results in Section 2 in detailed form. The reliability of $\eta_{h}$ will be proved in Section 3, with the efficiency in Section 4. In Section 5, we present some examples covered by our analysis, and provide a reliable and efficient computable upper bounds of $\mu_{h}\left(\gamma_{h}\right)$ for them.

\section{Notation AND MAin Results}

This section introduces necessary notations and states the main results of this paper.

2.1. Sobolev spaces and differential operators. We use the standard differential operators:

$$
\nabla r=(\partial r / \partial x, \partial r / \partial y), \quad \operatorname{Curl} p=(\partial p / \partial y,-\partial p / \partial x) .
$$

The linear Green strain $\varepsilon(\phi)=1 / 2\left[\nabla \phi+\nabla \phi^{T}\right]$ is the symmetric part of the gradient field $\nabla \boldsymbol{\phi}$. Given any $2 D$ vector function $\boldsymbol{\psi}=\left(\psi_{1}, \psi_{2}\right)$, its divergence reads $\operatorname{div} \boldsymbol{\psi}=\partial \psi_{1} / \partial x+\partial \psi_{2} / \partial y$. With the differential operator $\operatorname{rot} \boldsymbol{\psi}=\partial \psi_{2} / \partial x-\partial \psi_{1} / \partial y$ for a vector function $\boldsymbol{\psi}=\left(\psi_{1}, \psi_{2}\right)$, the space $H_{0}(\operatorname{rot}, \Omega)$ is defined as

$$
H_{0}(\operatorname{rot}, \Omega):=\left\{v \in L^{2}(\Omega)^{2} \mid \operatorname{rot} v \in L^{2}(\Omega) \text { and } v \cdot \boldsymbol{\tau}=0 \text { on } \partial \Omega\right\}
$$


endowed with the norm

$$
\|v\|_{H(\operatorname{rot}, \Omega)}:=\left(\|v\|_{L^{2}(\Omega)}^{2}+\|\operatorname{rot} v\|_{L^{2}(\Omega)}^{2}\right)^{1 / 2}
$$

The dual space for $H_{0}($ rot, $\Omega)$ reads

$$
H^{-1}(\operatorname{div}, \Omega):=\left\{v \in H^{-1}(\Omega)^{2} \mid \operatorname{div} v \in H^{-1}(\Omega)\right\},
$$

with the norm

$$
\|v\|_{H^{-1}(\operatorname{div}, \Omega)}:=\left(\|v\|_{H^{-1}(\Omega)}^{2}+\|\operatorname{div} v\|_{H^{-1}(\Omega)}^{2}\right)^{1 / 2} .
$$

We define the following mesh dependent norm, for any $(\boldsymbol{\psi}, v) \in H_{0}^{1}(\Omega)^{2} \times H_{0}^{1}(\Omega)$,

$$
\|(\boldsymbol{\psi}, v)\|_{1, h}^{2}=\|\nabla \boldsymbol{\psi}\|_{L^{2}(\Omega)}^{2}+\sum_{K \in \mathcal{T}_{h}} \frac{1}{t^{2}+h_{K}^{2}}\|\nabla v-\boldsymbol{\psi}\|_{L^{2}(K)}^{2} .
$$

For any functional $F$ over $H_{0}^{1}(\Omega)^{2} \times H_{0}^{1}(\Omega)$, we define its dual norm with respect to the norm (2.1) by

$$
\|F\|_{-1, h}=\sup _{(\boldsymbol{\psi}, v) \in H_{0}^{1}(\Omega)^{3} \backslash\{0\}} \frac{F(\boldsymbol{\psi}, v)}{\|(\boldsymbol{\psi}, v)\|_{1, h}} .
$$

The linear operator $\mathcal{C}$ is defined by

$$
\mathcal{C} \tau:=\frac{E}{12\left(1-\nu^{2}\right)}[(1-\nu) \tau+\nu \operatorname{tr}(\tau) I]
$$

for all $2 \times 2$ symmetric matrices. Here and throughout this paper, $t$ denotes the plate thickness with the shear modulus $\lambda=E \kappa / 2(1+\nu)$, the Young's modulus $E$, the Poisson ratio $\nu$, and the shear correction factor $\kappa$. The bilinear form $a(\boldsymbol{\phi}, \boldsymbol{\psi})$ is defined as

$$
a(\boldsymbol{\phi}, \boldsymbol{\psi})=(\mathcal{C} \varepsilon(\boldsymbol{\phi}), \varepsilon(\boldsymbol{\psi}))_{L^{2}(\Omega)} \text { for any } \boldsymbol{\phi}, \boldsymbol{\psi} \in \boldsymbol{\Theta}:=H_{0}^{1}(\Omega)^{2}
$$

which gives rise to the energy norm

$$
\|\boldsymbol{\psi}\|_{\mathcal{C}}^{2}:=a(\boldsymbol{\psi}, \boldsymbol{\psi}) \text { for any } \boldsymbol{\psi} \in \boldsymbol{\Theta}
$$

2.2. Triangulations and discrete spaces. Suppose that the closure $\bar{\Omega}$ is covered exactly by a regular triangulation $\mathcal{T}_{h}$ of $\bar{\Omega}$ into (closed) triangles or quadrilaterals in $2 D$ or other unions of simplices, that is,

$$
\bar{\Omega}=\bigcup \mathcal{T}_{h} \quad \text { and } \quad\left|K_{1} \cap K_{2}\right|=0 \quad \text { for } K_{1}, K_{2} \in \mathcal{T}_{h} \quad \text { with } K_{1} \neq K_{2},
$$

where $|\cdot|$ denotes the volume (as well as the length of an edge and the modulus of a vector etc. when there is no real risk of confusion). Let $\mathcal{E}$ denote the set of all edges in $\mathcal{T}_{h}$ with $\mathcal{E}(\Omega)$ the set of interior edges, and $\mathcal{N}(\Omega)$ the set of interior nodes. The set of edges of the element $K$ is denoted by $\mathcal{E}(K)$. By $h_{K}$ we denote the diameter of the element $K \in \mathcal{T}_{h}$. Also, we denote by $\overline{\omega_{K}}$ the union of elements $K^{\prime} \in \mathcal{T}_{h}$ that share an edge with $K$, and by $\overline{\omega_{E}}$ the union of elements having in common the edge $E$. Given any edge $E \in \mathcal{E}(\Omega)$ with length $h_{E}=|E|$ we assign one fixed unit normal $\nu_{E}:=\left(\nu_{1}, \nu_{2}\right)$ and tangential vector $\tau_{E}:=\left(-\nu_{2}, \nu_{1}\right)$. For $E$ on the boundary we choose the unit outward normal $\nu_{E}=\nu$ to $\Omega$. Once $\nu_{E}$ and $\tau_{E}$ have been fixed on $E$, in relation to $\nu_{E}$, one defines the elements $K_{-} \in \mathcal{T}_{h}$ and $K_{+} \in \mathcal{T}_{h}$, with $E=K_{+} \cap K_{-}$and $\overline{\omega_{E}}=K_{+} \cup K_{-}$. Given $E \in \mathcal{E}(\Omega)$ and some $\mathbb{R}^{d}$-valued function $v$ defined in $\Omega$, with $d=1,2$, we denote by $[v]:=\left.\left(\left.v\right|_{K_{+}}\right)\right|_{E}-\left.\left(\left.v\right|_{K_{-}}\right)\right|_{E}$ the jump of $v$ across $E$. 
Let $\hat{K}$ be a reference element. In the case of triangles $\hat{K}:=\left\{(\xi, \eta) \in \mathbb{R}^{2}: 0 \leq\right.$ $\xi \leq 1,0 \leq \eta \leq 1-\xi\}$, and quadrilaterals $\hat{K}:=[-1,1]^{2}$. The invertible linear (resp. bilinear) transformation $\hat{K} \rightarrow K$ is denoted by $F_{K}$ for any triangle (resp. quadrilateral) $K \in \mathcal{T}_{h}$ with the Jacobian matrix $D F_{K}$ and its determinant $J_{K}$.

Let $S_{0}^{1}\left(\mathcal{T}_{h}\right)$ denote the lowest order conforming finite element space over $\mathcal{T}_{h}$ which reads

$$
S_{0}^{1}\left(\mathcal{T}_{h}\right):=\left\{v \in H_{0}^{1}(\Omega): \forall K \in \mathcal{T}_{h},\left.v\right|_{K} \circ F_{K} \in Q_{1}(\hat{K})\right\} .
$$

Given any nonnegative integer $k$, the space $Q_{k}(\omega)$ consists of polynomials of total degree at most $k$ defined over $\omega$ in case $\omega=K$ is a triangle whereas it denotes polynomials of degree at most $k$ in each variable in case $K$ is a quadrilateral.

With the first order conforming finite element space $S_{0}^{1}\left(\mathcal{T}_{h}\right)$, we consider the Clément-type interpolation operator or any other regularized conforming finite element approximation operator $\mathcal{J}: H_{0}^{1}(\Omega) \mapsto S_{0}^{1}\left(\mathcal{T}_{h}\right)$ with the properties

$$
\begin{aligned}
& \|\nabla \mathcal{J} \varphi\|_{L^{2}(K)}+\left\|h_{K}^{-1}(\varphi-\mathcal{J} \varphi)\right\|_{L^{2}(K)} \lesssim\|\nabla \varphi\|_{L^{2}\left(\omega_{K}\right)} \text { and } \\
& \left\|h_{E}^{-1 / 2}(\varphi-\mathcal{J} \varphi)\right\|_{L^{2}(E)} \lesssim\|\nabla \varphi\|_{L^{2}\left(\omega_{K}\right)}
\end{aligned}
$$

for all $K \in \mathcal{T}_{h}, E \in \mathcal{E}(K)$, and $\varphi \in H_{0}^{1}(\Omega)$. The existence of such operators is guaranteed, for instance, in [25, 41, 20, 19].

Given $g \in L^{2}(\Omega)$, let $g_{h} \in Q_{k}\left(\mathcal{T}_{h}\right)$ denote its projection on the (possibly discontinuous) piecewise polynomial space of degree $k$ with respect to $\mathcal{T}_{h}$. We refer to $\operatorname{osc}(g)$ as oscillation of $g$

$$
\operatorname{osc}^{2}(g):=\sum_{K \in \mathcal{T}_{h}}\left(h_{K}^{2}+t^{2}\right) h_{K}^{2} \min _{g_{k} \in Q_{k}(K)}\left\|g-g_{k}\right\|_{L^{2}(K)}^{2} .
$$

2.3. Main results. The main results concern the reliability and efficiency of $\eta_{h}$. It is stressed that $\eta_{h}$ is the first a posteriori error estimator which estimates an error norm in which a priori convergence is guaranteed.

Theorem 2.1 (reliability). Suppose that $\boldsymbol{\Theta}_{h}, W_{h}, \boldsymbol{\Gamma}_{h}$ along with the reduction integration operator $\boldsymbol{R}_{h}$ satisfy $(\underline{\mathrm{H}})$ and $\left(S_{0}^{1}\left(\mathcal{T}_{h}\right)\right)^{2} \subset \mathbf{\Theta}_{h}$. Then, the estimator $\eta_{h}$ is reliable in the sense that

$$
\begin{gathered}
\left\|\boldsymbol{\phi}-\phi_{h}\right\|_{H^{1}(\Omega)}^{2}+\left\|\omega-\omega_{h}\right\|_{H^{1}(\Omega)}^{2}+t^{2}\left\|\gamma-\gamma_{h}\right\|_{L^{2}(\Omega)}^{2} \\
\quad+t^{4}\left\|\operatorname{rot}\left(\gamma-\gamma_{h}\right)\right\|_{L^{2}(\Omega)}^{2}+\left\|\gamma-\gamma_{h}\right\|_{H^{-1}(\Omega)}^{2} \lesssim \eta_{h}^{2} .
\end{gathered}
$$

Theorem 2.2 (efficiency). Suppose that $\boldsymbol{\Theta}_{h}, W_{h}, \boldsymbol{\Gamma}_{h}$ along with the reduction integration operator $\boldsymbol{R}_{h}$ satisfy (프) and $\left(S_{0}^{1}\left(\mathcal{T}_{h}\right)\right)^{2} \subset \boldsymbol{\Theta}_{h}$. Then, the estimator $\eta_{h}$ is efficient such that

$$
\begin{aligned}
\eta_{h}^{2} \lesssim \| \phi & -\phi_{h}\left\|_{H^{1}(\Omega)}^{2}+\right\| \omega-\omega_{h}\left\|_{H^{1}(\Omega)}^{2}+t^{2}\right\| \boldsymbol{\gamma}-\gamma_{h} \|_{L^{2}(\Omega)}^{2} \\
& +t^{4}\left\|\operatorname{rot}\left(\boldsymbol{\gamma}-\boldsymbol{\gamma}_{h}\right)\right\|_{L^{2}(\Omega)}^{2}+\left\|\boldsymbol{\gamma}-\boldsymbol{\gamma}_{h}\right\|_{H^{-1}(\Omega)}^{2}+\operatorname{osc}(g)^{2} .
\end{aligned}
$$

\section{Proof of Reliability}

This section is devoted to the proof of Theorem 2.1 which is divided into six steps. 
3.1. Splitting of $\left(I-\mathbf{R}_{h}\right) \phi_{h}$. Assume with (H) that $\left(\boldsymbol{R}_{h}-I\right) \phi_{h} \in H_{0}(\operatorname{rot}, \Omega)$. Then there exist $w \in H_{0}^{1}(\Omega)$ and $\boldsymbol{\beta} \in H_{0}^{1}(\Omega)^{2}$ with

$$
\begin{aligned}
\left(\boldsymbol{R}_{h}-I\right) \phi_{h} & =\nabla w-\boldsymbol{\beta} \text { and } \\
\|w\|_{H^{1}(\Omega)}+\|\boldsymbol{\beta}\|_{H^{1}(\Omega)} & \lesssim\left\|\left(\boldsymbol{R}_{h}-I\right) \phi_{h}\right\|_{H(\operatorname{rot}, \Omega)} .
\end{aligned}
$$

The proof of (3.1)-(3.2) can be found in Lemma 3.2 on Page 298 of [17.

3.2. Error representation formula. The residuals $\operatorname{Res}_{1}(\cdot)$ and $\operatorname{Res}_{2}(\cdot)$ are defined by

$$
\begin{aligned}
\operatorname{Res}_{1}(v) & =(g, v)_{L^{2}(\Omega)}-\left(\boldsymbol{\gamma}_{h}, \nabla v\right)_{L^{2}(\Omega)} \quad \text { for any } v \in H_{0}^{1}(\Omega), \\
\operatorname{Res}_{2}(\boldsymbol{\psi}) & =-a\left(\phi_{h}, \boldsymbol{\psi}\right)+\left(\boldsymbol{\gamma}_{h}, \boldsymbol{\psi}\right)_{L^{2}(\Omega)} \quad \text { for any } \boldsymbol{\psi} \in H_{0}^{1}(\Omega)^{2} .
\end{aligned}
$$

Notice that (1.1)-1.3) imply

$$
\begin{aligned}
& \left(\boldsymbol{\gamma}-\gamma_{h},\left(\boldsymbol{R}_{h}-I\right) \phi_{h}\right)_{L^{2}(\Omega)}=\left(\boldsymbol{\gamma}-\gamma_{h}, \nabla w-\boldsymbol{\beta}\right)_{L^{2}(\Omega)} \\
& =(g, w)_{L^{2}(\Omega)}-a(\boldsymbol{\phi}, \boldsymbol{\beta})-\left(\gamma_{h}, \nabla w-\boldsymbol{\beta}\right)_{L^{2}(\Omega)} \\
& =-a\left(\boldsymbol{\phi}-\boldsymbol{\phi}_{h}, \boldsymbol{\beta}\right)+(g, w)_{L^{2}(\Omega)}-a\left(\boldsymbol{\phi}_{h}, \boldsymbol{\beta}\right)-\left(\gamma_{h}, \nabla w-\boldsymbol{\beta}\right)_{L^{2}(\Omega)} .
\end{aligned}
$$

Therefore,

$$
\begin{aligned}
\left\|\boldsymbol{\phi}-\boldsymbol{\phi}_{h}\right\|_{\mathcal{C}}^{2}+\lambda^{-1} t^{2}\left\|\boldsymbol{\gamma}-\gamma_{h}\right\|_{L^{2}(\Omega)}^{2} \\
=a\left(\boldsymbol{\phi}-\boldsymbol{\phi}_{h}, \boldsymbol{\phi}-\boldsymbol{\phi}_{h}\right)+\left(\boldsymbol{\gamma}-\gamma_{h},\left(\nabla \omega-\nabla \omega_{h}\right)-\left(\boldsymbol{\phi}-\boldsymbol{\phi}_{h}\right)\right)_{L^{2}(\Omega)} \\
\quad+\left(\boldsymbol{\gamma}-\gamma_{h},\left(\boldsymbol{R}_{h}-I\right) \boldsymbol{\phi}_{h}\right)_{L^{2}(\Omega)} \\
=\operatorname{Res}_{1}\left(\omega-\omega_{h}+w\right)+\operatorname{Res}_{2}\left(\boldsymbol{\phi}-\boldsymbol{\phi}_{h}+\boldsymbol{\beta}\right)-a\left(\boldsymbol{\phi}-\boldsymbol{\phi}_{h}, \boldsymbol{\beta}\right) .
\end{aligned}
$$

It follows from the definitions of $\gamma$ and $\gamma_{h}$ and (3.1) that

$$
\begin{aligned}
\gamma-\gamma_{h} & =\lambda t^{-2}\left(\nabla \omega-\nabla \omega_{h}-\boldsymbol{\phi}+\boldsymbol{R}_{h} \boldsymbol{\phi}_{h}\right) \\
& =\lambda t^{-2}\left(\nabla \omega-\nabla \omega_{h}-\boldsymbol{\phi}+\boldsymbol{\phi}_{h}+\boldsymbol{R}_{h} \boldsymbol{\phi}_{h}-\boldsymbol{\phi}_{h}\right) \\
& =\lambda t^{-2}\left(\nabla \omega-\nabla \omega_{h}-\boldsymbol{\phi}+\boldsymbol{\phi}_{h}+\nabla w-\boldsymbol{\beta}\right) .
\end{aligned}
$$

Recalling that $\left\|\boldsymbol{\phi}-\boldsymbol{\phi}_{h}+\boldsymbol{\beta}\right\|_{\mathcal{C}}^{2}=a\left(\boldsymbol{\phi}-\boldsymbol{\phi}_{h}+\boldsymbol{\beta}, \boldsymbol{\phi}-\boldsymbol{\phi}_{h}+\boldsymbol{\beta}\right)$, we deduce

$$
\begin{aligned}
\frac{1}{2} & \left\|\boldsymbol{\phi}-\boldsymbol{\phi}_{h}+\boldsymbol{\beta}\right\|_{\mathcal{C}}^{2}+\frac{1}{2}\left\|\boldsymbol{\phi}-\boldsymbol{\phi}_{h}\right\|_{\mathcal{C}}^{2}+\frac{1}{2} \lambda^{-1} t^{2}\left\|\boldsymbol{\gamma}-\gamma_{h}\right\|_{L^{2}(\Omega)}^{2} \\
& +\frac{1}{2} \sum_{K \in \mathcal{T}_{h}} \frac{\lambda}{t^{2}+h_{K}^{2}}\left\|\nabla\left(\omega-\omega_{h}+w\right)-\left(\boldsymbol{\phi}-\boldsymbol{\phi}_{h}+\boldsymbol{\beta}\right)\right\|_{L^{2}(K)}^{2} \\
\leq & \left\|\boldsymbol{\phi}-\boldsymbol{\phi}_{h}\right\|_{\mathcal{C}}^{2}+\lambda^{-1} t^{2}\left\|\boldsymbol{\gamma}-\gamma_{h}\right\|_{L^{2}(\Omega)}^{2}+\frac{1}{2}\|\boldsymbol{\beta}\|_{\mathcal{C}}^{2}+a\left(\boldsymbol{\phi}-\boldsymbol{\phi}_{h}, \boldsymbol{\beta}\right) \\
= & \operatorname{Res}_{1}\left(\omega-\omega_{h}+w\right)+\operatorname{Res}_{2}\left(\boldsymbol{\phi}-\boldsymbol{\phi}_{h}+\boldsymbol{\beta}\right)-a\left(\boldsymbol{\phi}-\boldsymbol{\phi}_{h}, \boldsymbol{\beta}\right) \\
& +\frac{1}{2}\|\boldsymbol{\beta}\|_{\mathcal{C}}^{2}+a\left(\boldsymbol{\phi}-\boldsymbol{\phi}_{h}, \boldsymbol{\beta}\right) \\
= & \operatorname{Res}_{1}\left(\omega-\omega_{h}+w\right)+\operatorname{Res}_{2}\left(\boldsymbol{\phi}-\boldsymbol{\phi}_{h}+\boldsymbol{\beta}\right)+\frac{1}{2}\|\boldsymbol{\beta}\|_{\mathcal{C}}^{2} .
\end{aligned}
$$

3.3. Estimate for $\lambda^{-1} t^{2}\left\|\operatorname{rot}\left(\gamma-\gamma_{h}\right)\right\|$. Since

$$
\lambda^{-1} t^{2} \operatorname{rot}\left(\gamma-\gamma_{h}\right)=-\operatorname{rot}\left(\phi-\phi_{h}\right)-\operatorname{rot}\left(\phi_{h}-\boldsymbol{R}_{h} \phi_{h}\right),
$$

it holds that

$$
\lambda^{-1} t^{2}\left\|\operatorname{rot}\left(\gamma-\gamma_{h}\right)\right\|_{L^{2}(\Omega)} \lesssim\left\|\phi-\phi_{h}\right\|_{H^{1}(\Omega)}+\left\|\operatorname{rot}\left(\phi_{h}-\boldsymbol{R}_{h} \phi_{h}\right)\right\|_{L^{2}(\Omega)} .
$$


3.4. Estimate for $\left\|\gamma-\gamma_{h}\right\|$. For any $\boldsymbol{\psi} \in \boldsymbol{\Theta}$, it holds that

$$
\left(\boldsymbol{\gamma}-\boldsymbol{\gamma}_{h}, \boldsymbol{\psi}\right)_{L^{2}(\Omega)}=a\left(\boldsymbol{\phi}-\boldsymbol{\phi}_{h}, \boldsymbol{\psi}\right)_{L^{2}(\Omega)}+a\left(\boldsymbol{\phi}_{h}, \boldsymbol{\psi}\right)-\left(\boldsymbol{\gamma}_{h}, \boldsymbol{\psi}\right) .
$$

This proves

$$
\left\|\boldsymbol{\gamma}-\gamma_{h}\right\|_{H^{-1}(\Omega)} \lesssim\left\|\operatorname{Res}_{2}\right\|_{H^{-1}(\Omega)}+\left\|\boldsymbol{\phi}-\phi_{h}\right\|_{H^{1}(\Omega)} .
$$

3.5. Abstract a posteriori error estimate. Suppose that $\boldsymbol{\Theta}_{h}, W_{h}, \boldsymbol{\Gamma}_{h}$ along with the reduction integration operator $\boldsymbol{R}_{h}$ satisfy $(\underline{H})$. Then,

$$
\begin{aligned}
& \left\|\boldsymbol{\phi}-\boldsymbol{\phi}_{h}\right\|_{H^{1}(\Omega)}^{2}+\left\|\omega-\omega_{h}\right\|_{H^{1}(\Omega)}^{2}+t^{2}\left\|\boldsymbol{\gamma}-\gamma_{h}\right\|_{L^{2}(\Omega)}^{2} \\
& \quad+t^{4}\left\|\operatorname{rot}\left(\boldsymbol{\gamma}-\gamma_{h}\right)\right\|_{L^{2}(\Omega)}^{2}+\left\|\boldsymbol{\gamma}-\gamma_{h}\right\|_{H^{-1}(\Omega)}^{2} \\
& \lesssim\|\| \operatorname{Res}_{1}\left\|_{-1, h}^{2}+\right\| \operatorname{Res}_{2}\left\|_{H^{-1}(\Omega)}^{2}+\right\| \boldsymbol{\phi}_{h}-\boldsymbol{R}_{h} \boldsymbol{\phi}_{h} \|_{H(\operatorname{rot}, \Omega)}^{2} .
\end{aligned}
$$

In fact, given any $0<\varepsilon<1 / 2$, and $0<\alpha<1<t^{-1}$, it holds that

$$
\begin{aligned}
\lambda^{-1} & t^{2}\left\|\boldsymbol{\gamma}-\boldsymbol{\gamma}_{h}\right\|_{L^{2}(\Omega)}^{2} \\
= & \lambda\left(t^{-2}-\alpha^{2}\right)\left\|\nabla\left(\omega-\omega_{h}\right)-\left(\boldsymbol{\phi}-\boldsymbol{R}_{h} \boldsymbol{\phi}_{h}\right)\right\|_{L^{2}(\Omega)}^{2} \\
& +\lambda \alpha^{2}\left(\left\|\nabla\left(\omega-\omega_{h}\right)\right\|_{L^{2}(\Omega)}^{2}+\left\|\boldsymbol{\phi}-\boldsymbol{\phi}_{h}\right\|_{L^{2}(\Omega)}^{2}\right) \\
& +\lambda \alpha^{2}\left\|\boldsymbol{\phi}_{h}-\boldsymbol{R}_{h} \boldsymbol{\phi}_{h}\right\|_{L^{2}(\Omega)}^{2}+2 \lambda \alpha^{2}\left(\boldsymbol{\phi}-\boldsymbol{\phi}_{h}, \boldsymbol{\phi}_{h}-\boldsymbol{R}_{h} \boldsymbol{\phi}_{h}\right)_{L^{2}(\Omega)} \\
& -2 \lambda \alpha^{2}\left(\nabla\left(\omega-\omega_{h}\right), \boldsymbol{\phi}-\boldsymbol{\phi}_{h}\right)_{L^{2}(\Omega)}-2 \lambda \alpha^{2}\left(\nabla\left(\omega-\omega_{h}\right), \phi_{h}-\boldsymbol{R}_{h} \boldsymbol{\phi}_{h}\right)_{L^{2}(\Omega)} .
\end{aligned}
$$

Some Young inequalities prove

$$
\begin{aligned}
& \lambda\left(t^{-2}-\alpha^{2}\right)\left\|\nabla\left(\omega-\omega_{h}\right)-\left(\boldsymbol{\phi}-\boldsymbol{R}_{h} \boldsymbol{\phi}_{h}\right)\right\|_{L^{2}(\Omega)}^{2}+\lambda \alpha^{2}(1-2 \varepsilon)\left\|\nabla\left(\omega-\omega_{h}\right)\right\|_{L^{2}(\Omega)}^{2} \\
& \leq \lambda^{-1} t^{2}\left\|\boldsymbol{\gamma}-\gamma_{h}\right\|_{L^{2}(\Omega)}^{2}-\lambda \alpha^{2}\left(1-\frac{2}{\varepsilon}\right)\left\|\boldsymbol{\phi}_{h}-\boldsymbol{R}_{h} \boldsymbol{\phi}_{h}\right\|_{L^{2}(\Omega)}^{2} \\
& \quad-\lambda \alpha^{2}\left(1-\frac{1}{\varepsilon}-\varepsilon\right)\left\|\boldsymbol{\phi}-\boldsymbol{\phi}_{h}\right\|_{L^{2}(\Omega)}^{2} .
\end{aligned}
$$

An appropriate choice of $\alpha$ and $\varepsilon$ and the Korn and Young inequalities, the preceding inequality, (3.3), and (3.2) yield

$$
\begin{aligned}
& \left\|\boldsymbol{\phi}-\boldsymbol{\phi}_{h}\right\|_{H^{1}(\Omega)}+\left\|\omega-\omega_{h}\right\|_{H^{1}(\Omega)}+t\left\|\boldsymbol{\gamma}-\boldsymbol{\gamma}_{h}\right\|_{L^{2}(\Omega)} \\
& \lesssim\|\| \operatorname{Res}_{1}\left\|_{-1, h}+\right\| \operatorname{Res}_{2}\left\|_{H^{-1}(\Omega)}+\right\| \boldsymbol{\phi}_{h}-\boldsymbol{R}_{h} \boldsymbol{\phi}_{h} \|_{H(\mathrm{rot}, \Omega)} .
\end{aligned}
$$

Together with Subsections 3.3 and 3.4 this proves the assertion (3.5).

3.6. Refined approximation of the Clément interpolation. Following the idea of [22, we have the refined approximation property for the Clément interpolation. Given any $v \in H_{0}^{1}(\Omega)$, set $v_{h}=\mathcal{J} v$, such that there holds, for any $K \in \mathcal{T}_{h}$ and $\boldsymbol{\psi} \in H_{0}^{1}(\Omega)^{2}$, that

$$
h_{K}^{-1}\left\|v-v_{h}\right\|_{L^{2}(K)}+h_{E}^{-1 / 2}\left\|v-v_{h}\right\|_{L^{2}(E)} \lesssim\|\nabla v-\boldsymbol{\psi}\|_{L^{2}\left(\omega_{K}\right)}+h_{K}\|\nabla \boldsymbol{\psi}\|_{L^{2}\left(\omega_{K}\right)}
$$

in the sense of Remark 3.1, for any $E \subset \partial K$. To prove (3.6), suppose in the first case that one vertex of $K$ belongs to the boundary. We assume that the intersection of $\partial \omega_{K}$ with $\partial \Omega$ contains at least one edge. So a Friedrichs inequality shows

$$
\|\boldsymbol{\psi}\|_{L^{2}\left(\omega_{K}\right)} \lesssim h_{K}\|\nabla \boldsymbol{\psi}\|_{L^{2}\left(\omega_{K}\right)}
$$

Together with a triangle inequality this yields (3.6). 
Remark 3.1. In case $\partial \omega_{K}$ does not contain one edge, one can enlarge $\omega_{K}$ to $\omega_{K}^{\prime}$ so that $\partial \omega_{K}^{\prime}$ contains one edge. Then (3.6) holds for $\omega_{K}^{\prime}$. The analysis herein is equally valid for this small modification.

In the second case, the vertices of $K$ are interior nodes and so $\left.\left(v-v_{h}\right)\right|_{K}$ remains the same if we change $v$ to $v-z$ for an affine function $z$ on $\omega_{K}$ when we change $v_{h}$ accordingly; the Clément approximation operator locally preserves affine functions. We choose the constant vector $A:=\nabla z$ as the integral mean of $\boldsymbol{\psi}$ on $\omega_{K}$. As a consequence, (2.6)-(2.7) can be recast as

$$
h_{K}^{-1}\left\|v-v_{h}\right\|_{L^{2}(K)}+h_{E}^{-1 / 2}\left\|v-v_{h}\right\|_{L^{2}(E)} \lesssim\|\nabla v-A\|_{L^{2}\left(\omega_{K}\right)} .
$$

Hence a Poincaré inequality shows

$$
\|\boldsymbol{\psi}-A\|_{L^{2}\left(\omega_{K}\right)} \lesssim h_{K}\|\nabla \boldsymbol{\psi}\|_{L^{2}\left(\omega_{K}\right)}
$$

This concludes the proof of (3.6).

3.7. Proof of reliability. The proof is based on (3.5)-(3.6) of Subsections 3.5 and

3.6. For any $v \in H_{0}^{1}(\Omega)$, let $v_{h}=\mathcal{J} v$ in (1.3), and $\boldsymbol{\psi} \in H_{0}^{1}(\Omega)^{2}$ as in Subsection 3.6. An integration by parts shows

$$
\begin{aligned}
\operatorname{Res}_{1}(v)= & \operatorname{Res}_{1}\left(v-v_{h}\right) \lesssim\left(\sum_{K \in \mathcal{T}_{h}} h_{K}^{2}\left(t^{2}+h_{K}^{2}\right)\left\|g+\operatorname{div} \gamma_{h}\right\|_{L^{2}(K)}^{2}\right. \\
& \left.+\sum_{E \in \mathcal{E}(\Omega)} h_{E}\left(t^{2}+h_{E}^{2}\right)\left\|\left[\boldsymbol{\gamma}_{h}\right] \cdot \nu_{E}\right\|_{L^{2}(E)}^{2}\right)^{1 / 2}\|(\boldsymbol{\psi}, v)\|_{1, h} .
\end{aligned}
$$

Let $\psi_{h}=\mathcal{J} \psi$ with (1.3), then

$$
\operatorname{Res}_{2}(\boldsymbol{\psi})=-a\left(\boldsymbol{\phi}_{h}, \boldsymbol{\psi}-\boldsymbol{\psi}_{h}\right)+\left(\boldsymbol{\gamma}_{h}, \boldsymbol{\psi}-\boldsymbol{\psi}_{h}\right)+\left(\boldsymbol{\gamma}_{h}, \boldsymbol{\psi}_{h}-\boldsymbol{R}_{h} \boldsymbol{\psi}_{h}\right) .
$$

An integration by parts and (2.6)-(2.7) imply

$$
\begin{aligned}
\operatorname{Res}_{2}(\boldsymbol{\psi}) \lesssim & \left(\sum_{K \in \mathcal{T}_{h}} h_{K}^{2}\left\|\operatorname{div} \mathcal{C} \varepsilon\left(\boldsymbol{\phi}_{h}\right)+\gamma_{h}\right\|_{L^{2}(K)}^{2}\right. \\
& \left.+\sum_{E \in \mathcal{E}(\Omega)} h_{E}\left\|\left[\mathcal{C} \varepsilon\left(\phi_{h}\right)\right] \cdot \nu_{E}\right\|_{L^{2}(E)}^{2}\right)^{1 / 2}\|\boldsymbol{\psi}\|_{H^{1}(\Omega)} \\
& +\sup _{\boldsymbol{\psi}_{h} \in\left(S_{0}^{1}\left(\mathcal{T}_{h}\right)\right)^{2} \backslash\{0\}} \frac{\left(\boldsymbol{\gamma}_{h},\left(I-\boldsymbol{R}_{h}\right) \boldsymbol{\psi}_{h}\right)_{L^{2}(\Omega)}}{\|\boldsymbol{\psi}\|_{H^{1}(\Omega)}}
\end{aligned}
$$

Remark 3.2. In this paper, we use the norm || $\operatorname{Res}_{1}|\||_{-1, h}$ instead of $\left\|\operatorname{Res}_{1}\right\|_{H^{-1}(\Omega)}$ to get a factor $t^{2}+h_{E}^{2}$ (resp. $t^{2}+h_{K}^{2}$ ), which is essential for the efficiency of the estimator in the next section.

\section{EFFICIENCY OF $\eta$}

This section is devoted to the proof of the efficiency of $\eta_{h}$ from Theorem 2.2 The contributions are analyzed separately and even locally. 
4.1. Efficiency of $\left\|g+\operatorname{div} \gamma_{h}\right\|$. We choose

$$
w_{K}=B_{K}^{2}\left(g_{h}+\operatorname{div} \gamma_{h}\right) .
$$

Here and throughout this section, $g_{h}=\Pi_{h} g$, and $B_{K}$ denotes the standard element bubble function with the following properties [47]:

$$
\begin{aligned}
& \operatorname{supp} B_{K} \subset K, \quad 0 \leq B_{K} \leq 1, \quad \max _{x \in K} B_{K}=1, \\
& \int_{K} B_{K} d x \approx h_{K}^{2}, \text { and }\left\|\nabla B_{K}\right\|_{L^{2}(K)} \lesssim h_{K}^{-1}\left\|B_{K}\right\|_{L^{2}(K)} .
\end{aligned}
$$

An integration by parts shows

$$
\begin{aligned}
& \left\|g_{h}+\operatorname{div} \gamma_{h}\right\|_{L^{2}(K)}^{2} \lesssim \int_{K}\left(g_{h}+\operatorname{div} \gamma_{h}\right) w_{K} d x d y \\
& =\left(g_{h}, w_{K}\right)_{L^{2}(K)}-\left(\gamma_{h}, \nabla w_{K}\right)_{L^{2}(K)} \\
& =\left(\gamma-\gamma_{h}, \nabla w_{K}\right)_{L^{2}(K)}+\left(g_{h}-g, w_{K}\right)_{L^{2}(K)} .
\end{aligned}
$$

The sum over all elements leads to

$$
\begin{aligned}
& \sum_{K \in \mathcal{T}_{h}} h_{K}^{2}\left(h_{K}^{2}+t^{2}\right)\left\|g_{h}+\operatorname{div} \gamma_{h}\right\|_{L^{2}(K)}^{2} \\
& \lesssim \sum_{K \in \mathcal{T}_{h}} t\left\|\gamma-\gamma_{h}\right\|_{L^{2}(K)} t h_{K}^{2}\left\|\nabla w_{K}\right\|_{L^{2}(K)} \\
& \quad+\left\|\gamma-\gamma_{h}\right\|_{H^{-1}(\Omega)}\left\|\sum_{K \in \mathcal{T}_{h}} h_{K}^{4} \nabla w_{K}\right\|_{H^{1}(\Omega)} \\
& \quad+\sum_{K \in \mathcal{T}_{h}}\left(h_{k}^{2}+t^{2}\right)^{1 / 2} h_{K}\left\|g_{h}-g\right\|_{L^{2}(K)}\left\|h_{K}\left(h_{k}^{2}+t^{2}\right)^{1 / 2} w_{K}\right\|_{L^{2}(K)} .
\end{aligned}
$$

An elementwise inverse estimate yields

$$
\left\|\nabla w_{K}\right\|_{L^{2}(K)}+h_{K}\left\|\nabla w_{K}\right\|_{H^{1}(K)} \lesssim h_{K}^{-1}\left\|g_{h}+\operatorname{div} \gamma_{h}\right\|_{L^{2}(K)} .
$$

This implies

$$
\begin{aligned}
& \sum_{K \in \mathcal{T}_{h}} h_{K}^{2}\left(h_{K}^{2}+t^{2}\right)\left\|g_{h}+\operatorname{div} \gamma_{h}\right\|_{L^{2}(K)}^{2} \\
& \lesssim t^{2}\left\|\boldsymbol{\gamma}-\gamma_{h}\right\|_{L^{2}(\Omega)}^{2}+\left\|\boldsymbol{\gamma}-\gamma_{h}\right\|_{H^{-1}(\Omega)}^{2}+\sum_{K \in \mathcal{T}_{h}}\left(h_{k}^{2}+t^{2}\right) h_{K}^{2}\left\|g_{h}-g\right\|_{L^{2}(K)}^{2} .
\end{aligned}
$$

4.2. Efficiency of $\left\|\operatorname{div} \mathcal{C} \varepsilon\left(\phi_{h}\right)+\gamma_{h}\right\|$. Recall $B_{K}$ from the previous section, and set

$$
\boldsymbol{\beta}_{K}:=B_{K}\left(\operatorname{div} \mathcal{C} \varepsilon\left(\phi_{h}\right)+\gamma_{h}\right) \text {. }
$$

An integration by parts leads to

$$
\begin{aligned}
\left\|\operatorname{div} \mathcal{C} \varepsilon\left(\boldsymbol{\phi}_{h}\right)+\boldsymbol{\gamma}_{h}\right\|_{L^{2}(K)}^{2} & \lesssim\left(\operatorname{div} \mathcal{C} \varepsilon\left(\boldsymbol{\phi}_{h}\right)+\boldsymbol{\gamma}_{h}, \boldsymbol{\beta}_{K}\right)_{L^{2}(K)} \\
& =-\left(\mathcal{C} \varepsilon\left(\boldsymbol{\phi}_{h}\right), \varepsilon\left(\boldsymbol{\beta}_{K}\right)\right)_{L^{2}(K)}+\left(\boldsymbol{\gamma}_{h}, \boldsymbol{\beta}_{K}\right)_{L^{2}(K)} \\
& =-\left(\mathcal{C} \varepsilon\left(\boldsymbol{\phi}_{h}-\boldsymbol{\phi}\right), \varepsilon\left(\boldsymbol{\beta}_{K}\right)\right)+\left(\boldsymbol{\gamma}_{h}-\boldsymbol{\gamma}, \boldsymbol{\beta}_{K}\right)_{L^{2}(K)}
\end{aligned}
$$

The arguments of Subsection 4.1 allow the proof of

$$
\sum_{K \in \mathcal{T}_{h}} h_{K}^{2}\left\|\operatorname{div} \mathcal{C} \varepsilon\left(\phi_{h}\right)+\gamma_{h}\right\|_{L^{2}(K)}^{2} \lesssim\left\|\phi_{h}-\phi\right\|_{H^{1}(\Omega)}^{2}+\left\|\gamma-\gamma_{h}\right\|_{H^{-1}(\Omega)}^{2} .
$$


4.3. Efficiency of $\left\|\left[\nu_{E} \cdot \gamma_{h}\right]\right\|$. Given any interior edge $E=\partial K_{+} \cap \partial K_{-}$, let $b_{E} \in$ $H_{0}^{2}\left(\omega_{E}\right)$ denote the edge bubble function from [47, and set

$$
w_{E}=b_{E}\left[\nu_{E} \cdot \gamma_{h}\right]
$$

One can prove $b_{E}$ satisfies the following properties:

$$
\begin{aligned}
& \operatorname{supp} b_{E}=\overline{\omega_{E}}, \quad 0 \leq b_{E} \leq 1=\max _{x \in E} b_{E}, \\
& \int_{\omega_{E}} b_{E} d x \approx h_{E}^{2} \text { and } \int_{E} b_{E} d s \approx h_{E}, \\
& \left\|\nabla b_{E}\right\|_{L^{2}\left(K_{ \pm}\right)} \lesssim h_{E}^{-1}\left\|b_{E}\right\|_{L^{2}\left(K_{ \pm}\right)},\left|\nabla b_{E}\right|_{H^{1}\left(\omega_{E}\right)} \lesssim h_{E}^{-2}\left\|b_{E}\right\|_{L^{2}\left(\omega_{E}\right)} .
\end{aligned}
$$

For any $E \in \mathcal{E}(\Omega)$, it holds that

$$
\begin{aligned}
\left\|\left[\nu_{E} \cdot \gamma_{h}\right]\right\|_{L^{2}(E)}^{2} & \lesssim\left(\left[\nu_{E} \cdot \gamma_{h}\right], w_{E}\right)_{L^{2}(E)} \\
& =\left(\operatorname{div} \gamma_{h}, w_{E}\right)_{L^{2}\left(\omega_{E}\right)}+\left(\gamma_{h}, \nabla w_{E}\right)_{L^{2}\left(\omega_{E}\right)} \\
& =\left(\operatorname{div} \gamma_{h}+g, w_{E}\right)_{L^{2}\left(\omega_{E}\right)}+\left(\gamma_{h}-\gamma, \nabla w_{E}\right)_{L^{2}\left(\omega_{E}\right)} .
\end{aligned}
$$

The sum over interior edges, the Cauchy inequality plus the shape regularity show

$$
\begin{aligned}
& \sum_{E \in \mathcal{E}(\Omega)} h_{E}\left(h_{E}^{2}+t^{2}\right)\left\|\left[\nu_{E} \cdot \gamma_{h}\right]\right\|_{L^{2}(E)}^{2} \\
& \lesssim \sum_{E \in \mathcal{E}(\Omega)}\left(\operatorname{div} \gamma_{h}+g, h_{E}\left(h_{E}^{2}+t^{2}\right) w_{E}\right)_{L^{2}\left(\omega_{E}\right)}+\left(\gamma_{h}-\gamma, \nabla h_{E}\left(h_{E}^{2}+t^{2}\right) w_{E}\right)_{L^{2}\left(\omega_{E}\right)} \\
& \lesssim\left(\sum_{K \in \mathcal{T}_{h}} h_{K}^{2}\left(h_{K}^{2}+t^{2}\right)\left\|\operatorname{div} \gamma_{h}+g\right\|_{L^{2}(K)}^{2}\right)^{1 / 2}\left(\sum_{E \in \mathcal{E}(\Omega)}\left(h_{E}^{2}+t^{2}\right)\left\|w_{E}\right\|_{L^{2}\left(\omega_{E}\right)}^{2}\right)^{1 / 2} \\
& \quad+\left\|\gamma_{h}-\gamma\right\|_{H^{-1}(\Omega)}\left\|\sum_{E \in \mathcal{E}(\Omega)} h_{E}^{3} \nabla w_{E}\right\|_{H^{1}(\Omega)} \\
& \quad+t\left\|\gamma_{h}-\gamma\right\|_{L^{2}(\Omega)}\left\|\sum_{E \in \mathcal{E}(\Omega)} t h_{E} \nabla w_{E}\right\|_{L^{2}(\Omega)}
\end{aligned}
$$

The elementwise inverse estimate implies

$$
\left\|\nabla w_{E}\right\|_{L^{2}\left(\omega_{E}\right)}+h_{E}\left|\nabla w_{E}\right|_{H^{1}\left(\omega_{E}\right)} \lesssim h_{E}^{-1 / 2}\left\|\left[\nu_{E} \cdot \gamma_{h}\right]\right\|_{L^{2}(E)} .
$$

Since $\sum_{E \in \mathcal{E}(\Omega)} h_{E}^{3} \nabla w_{E} \in H_{0}^{1}(\Omega)^{2}$, we use the finite overlapping of the supports of $\nabla w_{E}$ with $E \in \mathcal{E}(\Omega)$ and the above estimate to derive as

$$
\begin{aligned}
\left\|\sum_{E \in \mathcal{E}(\Omega)} h_{E}^{3} \nabla w_{E}\right\|_{H^{1}(\Omega)}^{2} & \lesssim \sum_{E \in \mathcal{E}(\Omega)}\left\|h_{E}^{3} \nabla w_{E}\right\|_{H^{1}\left(\omega_{E}\right)}^{2} \lesssim \sum_{E \in \mathcal{E}(\Omega)} h_{E}^{3}\left\|\left[\nu_{E} \cdot \gamma_{h}\right]\right\|_{L^{2}(E)}^{2} \\
& \leq \sum_{E \in \mathcal{E}(\Omega)} h_{E}\left(t^{2}+h_{E}^{2}\right)\left\|\left[\nu_{E} \cdot \gamma_{h}\right]\right\|_{L^{2}(E)}^{2} .
\end{aligned}
$$

A similar argument leads to

$$
\left\|\sum_{E \in \mathcal{E}(\Omega)} t h_{E} \nabla w_{E}\right\|_{L^{2}(\Omega)}^{2} \lesssim \sum_{E \in \mathcal{E}(\Omega)} h_{E}\left(t^{2}+h_{E}^{2}\right)\left\|\left[\nu_{E} \cdot \gamma_{h}\right]\right\|_{L^{2}(E)}^{2} .
$$


A patchwise Poincaré inequality gives

$$
\begin{aligned}
\sum_{E \in \mathcal{E}(\Omega)}\left(h_{E}^{2}+t^{2}\right)\left\|w_{E}\right\|_{L^{2}\left(\omega_{E}\right)}^{2} & \lesssim \sum_{E \in \mathcal{E}(\Omega)}\left(h_{E}^{2}+t^{2}\right) h_{E}^{2}\left\|\nabla w_{E}\right\|_{L^{2}\left(\omega_{E}\right)}^{2} \\
& \lesssim \sum_{E \in \mathcal{E}(\Omega)} h_{E}\left(t^{2}+h_{E}^{2}\right)\left\|\left[\nu_{E} \cdot \gamma_{h}\right]\right\|_{L^{2}(E)}^{2} .
\end{aligned}
$$

A summary of these estimates yields

$$
\begin{aligned}
& \sum_{E \in \mathcal{E}(\Omega)} h_{E}\left(h_{E}^{2}+t^{2}\right)\left\|\left[\nu_{E} \cdot \gamma_{h}\right]\right\|_{L^{2}(E)}^{2} \\
& \lesssim t^{2}\left\|\gamma-\gamma_{h}\right\|_{L^{2}(\Omega)}^{2}+\left\|\boldsymbol{\gamma}-\gamma_{h}\right\|_{H^{-1}(\Omega)}^{2}+\sum_{K \in \mathcal{T}_{h}}\left(h_{K}^{2}+t^{2}\right) h_{K}^{2}\left\|g_{h}-g\right\|_{L^{2}(K)}^{2} .
\end{aligned}
$$

Remark 4.1. Note that compared to the usual linear elliptic problem, the edge bubble $b_{E} \in H^{2}\left(\omega_{E}\right)$ is of high regularity for the proof of the efficiency of $\left\|\left[\nu_{E} \cdot \gamma_{h}\right]\right\|_{L^{2}(E)}$.

4.4. Efficiency of $\left\|\left[\nu_{E} \cdot \mathcal{C} \varepsilon\left(\phi_{h}\right)\right]\right\|$. For any $E \in \mathcal{E}(\Omega), B_{E} \in H_{0}^{1}\left(\omega_{E}\right)$ denote the usual edge bubble function [47] with

$$
\begin{aligned}
& \operatorname{supp} B_{E} \subset \omega_{E}, \quad 0 \leq B_{E} \leq 1, \quad \max _{x \in E} B_{E}=1, \\
& \int_{K_{ \pm}} B_{E} d x \approx \int_{\omega_{E}} B_{E} d x \approx h_{E}^{2}, \\
& \int_{E} B_{E} d s \approx h_{E}, \text { and }\left\|\nabla B_{E}\right\|_{L^{2}\left(\omega_{E}\right)} \lesssim h_{E}^{-1}\left\|B_{E}\right\|_{L^{2}\left(\omega_{E}\right)} .
\end{aligned}
$$

Set

Standard arguments verify

$$
\boldsymbol{\beta}_{E}=B_{E}\left[\nu_{E} \cdot \mathcal{C} \varepsilon\left(\phi_{h}\right)\right]
$$

$$
\begin{aligned}
\left\|\left[\nu_{E} \cdot \mathcal{C} \varepsilon\left(\phi_{h}\right)\right]\right\|_{L^{2}(E)}^{2} \lesssim & \left(\left[\nu_{E} \cdot \mathcal{C} \varepsilon\left(\phi_{h}\right)\right], \boldsymbol{\beta}_{E}\right)_{E} \\
= & \left(\operatorname{div} \mathcal{C} \varepsilon\left(\boldsymbol{\phi}_{h}\right)+\boldsymbol{\gamma}_{h}, \boldsymbol{\beta}_{E}\right)_{L^{2}\left(\omega_{E}\right)}+\left(\mathcal{C} \varepsilon\left(\boldsymbol{\phi}_{h}-\boldsymbol{\phi}\right), \varepsilon\left(\boldsymbol{\beta}_{E}\right)\right)_{L^{2}\left(\omega_{E}\right)} \\
& +\left(\boldsymbol{\gamma}-\boldsymbol{\gamma}_{h}, \boldsymbol{\beta}_{E}\right)_{L^{2}\left(\omega_{E}\right)} .
\end{aligned}
$$

This, (4.2), and an elementwise inverse estimate yield

$$
\sum_{E \in \mathcal{E}(\Omega)} h_{E}\left\|\left[\nu_{E} \cdot \mathcal{C} \varepsilon\left(\phi_{h}\right)\right]\right\|_{L^{2}(E)}^{2} \lesssim\left\|\gamma_{h}-\gamma\right\|_{H^{-1}(\Omega)}^{2}+\left\|\phi-\phi_{h}\right\|_{H^{1}(\Omega)}^{2} .
$$

4.5. Efficiency of $\left\|\phi_{h}-\mathbf{R}_{h} \phi_{h}\right\|$ and $\mu_{h}\left(\gamma_{h}\right)$. By the definitions of $\boldsymbol{\gamma}$, and $\gamma_{h}$,

$$
\phi_{h}-\boldsymbol{R}_{h} \boldsymbol{\phi}_{h}=-\lambda^{-1} t^{2}\left(\boldsymbol{\gamma}-\gamma_{h}\right)-\left(\boldsymbol{\phi}-\boldsymbol{\phi}_{h}\right)+\nabla\left(\omega-\omega_{h}\right) \text {. }
$$

Therefore,

$$
\begin{aligned}
\| \phi_{h}- & \boldsymbol{R}_{h} \phi_{h}\left\|_{H(\operatorname{rot}, \Omega)} \lesssim\right\| \nabla\left(\omega-\omega_{h}\right) \|_{L^{2}(\Omega)} \\
& +\left\|\phi-\phi_{h}\right\|_{H^{1}(\Omega)}+t^{2}\left\|\operatorname{rot}\left(\gamma-\gamma_{h}\right)\right\|_{L^{2}(\Omega)} .
\end{aligned}
$$

It remains to estimate the last term. For any $\boldsymbol{\psi}_{h} \in\left(S_{0}^{1}\left(\mathcal{T}_{h}\right)\right)^{2}$, it holds that

$$
\begin{aligned}
\left(\gamma_{h},\left(I-\boldsymbol{R}_{h}\right) \boldsymbol{\psi}_{h}\right) & =\left(\boldsymbol{\gamma}_{h}, \boldsymbol{\psi}_{h}\right)-\left(\boldsymbol{\gamma}_{h}, \boldsymbol{R}_{h} \boldsymbol{\psi}_{h}\right) \\
& =\left(\boldsymbol{\gamma}_{h}-\boldsymbol{\gamma}, \boldsymbol{\psi}_{h}\right)+a\left(\boldsymbol{\phi}_{h}-\boldsymbol{\phi}, \boldsymbol{\psi}_{h}\right) \\
& \lesssim\left(\left\|\boldsymbol{\gamma}-\gamma_{h}\right\|_{H^{-1}(\Omega)}+\left\|\boldsymbol{\phi}-\boldsymbol{\phi}_{h}\right\|_{H^{1}(\Omega)}\right)\left\|\boldsymbol{\psi}_{h}\right\|_{H^{1}(\Omega)}
\end{aligned}
$$


Consequently,

$$
\sup _{\boldsymbol{\psi}_{h} \in\left(S_{0}^{1}\left(\mathcal{T}_{h}\right)\right)^{2} \backslash\{0\}} \frac{\left(\gamma_{h},\left(I-\boldsymbol{R}_{h}\right) \boldsymbol{\psi}_{h}\right)_{L^{2}(\Omega)}}{\left\|\boldsymbol{\psi}_{h}\right\|_{H^{1}(\Omega)}} \lesssim\left\|\boldsymbol{\gamma}-\gamma_{h}\right\|_{H^{-1}(\Omega)}+\left\|\boldsymbol{\phi}-\boldsymbol{\phi}_{h}\right\|_{H^{1}(\Omega)} .
$$

\section{EXAmples}

This section presents a list of examples from Table 1 which allows the computable upper bound for $\mu_{h}\left(\gamma_{h}\right)$, namely

$$
\begin{aligned}
\mu_{h}\left(\gamma_{h}\right)^{2} & \lesssim \sum_{E \in \mathcal{E}(\Omega)} h_{E}\left(h_{E}^{2}+t^{2}\right)\left\|\left[\gamma_{h}\right] \cdot \nu_{E}\right\|_{L^{2}(E)}^{2} \\
& +\sum_{K \in \mathcal{T}_{h}} h_{K}^{2}\left(h_{K}^{2}+t^{2}\right)\left\|\operatorname{div} \gamma_{h}+g\right\|_{L^{2}(K)}^{2}+\text { h.o.t. }
\end{aligned}
$$

up to some computable high-order terms h.o.t. The previous section shows its efficiency.

Remark 5.1. The list of Table 1 is not comprehensive. In fact, we conjecture that all known conforming MITC elements could be analyzed in the present framework. (Only the triangular DL element has been considered with a different a posteriori error estimator in [36]; cf. Remark [5.2.) The present theory leads to a new reliable and efficient error control for all elements. Because the MITC procedure is used the condition ( $(\mathrm{H})$ is satisfied in all of those examples.

Remark 5.2. Compared to the a posterior error estimator of [36] for the DL element, the estimator $\eta_{h}$ here is different in two points. First the shear force norm is different; second the estimator for the shear force is different.

5.1. Conforming elements without reduction integration. The first example is the conforming (triangle or quadrilateral) element without reduction. Since $\boldsymbol{R}_{h}=$ $I, \mu_{h}\left(\gamma_{h}\right)=0$.

5.2. Triangle MITC elements. This subsection discusses triangle MITC elements from literature.

5.2.1. The MITC3 element. For a triangulation into triangular elements, let

$$
\begin{aligned}
& W_{h}:=\left\{v \in H_{0}^{1}(\Omega):\left.v\right|_{K} \in Q_{1}(K), K \in \mathcal{T}_{h}\right\}, \\
& \boldsymbol{\Theta}_{h}:=\left\{\boldsymbol{\beta} \in H_{0}^{1}(\Omega)^{2}:\left.\boldsymbol{\beta}\right|_{K} \in\left(Q_{1}(K)\right)^{2}, K \in \mathcal{T}_{h}\right\}, \\
& \boldsymbol{\Gamma}_{h}:=\left\{\boldsymbol{\sigma} \in H_{0}(\operatorname{rot}, \Omega):\left.\boldsymbol{\sigma}\right|_{K} \in\left(Q_{0}(K)\right)^{2} \oplus Q_{0}(K)(y,-x)^{T}, K \in \mathcal{T}_{h}\right\}
\end{aligned}
$$

and define the reduction operator $\boldsymbol{R}_{h}$ by

$$
\int_{E}\left(\boldsymbol{R}_{h} \boldsymbol{\sigma}-\boldsymbol{\sigma}\right) \cdot \boldsymbol{\tau}_{E} d s=0 \text { for every edge } E \text { of } K .
$$

This FEM is unstable and is not encouraged in practise. However, $(\underline{H})$ holds, and $\eta_{h}$ is a reliable and efficient a posteriori error estimator. With this choice, there exists $\psi \in H_{0}^{1}(\Omega)$ with $\left.\psi\right|_{K} \in Q_{2}(K)$ and

$$
\nabla \psi=\boldsymbol{\beta}-\boldsymbol{R}_{h} \boldsymbol{\beta} \text { for any } \boldsymbol{\beta} \in \boldsymbol{\Theta}_{h} .
$$

It is proved in [26, 38] that $\psi$ vanishes at all nodes. Consequently,

$$
\|\psi\|_{L^{2}(\partial K)} \lesssim h_{K}^{1 / 2}\|\nabla \psi\|_{L^{2}(K)} .
$$


Since $\operatorname{div} \gamma_{h}=0$, an integration by parts with (5.2) gives

$$
\begin{aligned}
\left(\gamma_{h}, \boldsymbol{\beta}-\boldsymbol{R}_{h} \boldsymbol{\beta}\right)_{L^{2}(\Omega)} \lesssim \sum_{E \in \mathcal{E}(\Omega)} h_{E}^{1 / 2}\left\|\left[\gamma_{h} \cdot \nu_{E}\right]\right\|_{L^{2}(E)}^{2}\left\|\boldsymbol{\beta}-\boldsymbol{R}_{h} \boldsymbol{\beta}\right\|_{L^{2}(K)} \\
\quad \lesssim\left(\sum_{E \in \mathcal{E}(\Omega)} h_{E}^{3}\left\|\left[\gamma_{h} \cdot \nu_{E}\right]\right\|_{L^{2}(E)}^{2}\right)^{1 / 2}\|\boldsymbol{\beta}\|_{H^{1}(\Omega)} \\
\quad \lesssim\left(\sum_{E \in \mathcal{E}(\Omega)} h_{E}\left(h_{E}^{2}+t^{2}\right)\left\|\left[\gamma_{h} \cdot \nu_{E}\right]\right\|_{L^{2}(E)}^{2}\right)^{1 / 2}\|\boldsymbol{\beta}\|_{H^{1}(\Omega)} \text { for any } \boldsymbol{\beta} \in\left(S_{0}^{1}\left(\mathcal{T}_{h}\right)\right)^{2} .
\end{aligned}
$$

This proves (5.1). In the second inequality we use the following approximation for the operator $\boldsymbol{R}_{h}$,

$$
\left\|\left(I-\boldsymbol{R}_{h}\right) \boldsymbol{\beta}\right\|_{L^{2}(K)} \lesssim h_{K}\|\boldsymbol{\beta}\|_{H^{1}(K)} \text { for any } \boldsymbol{\beta} \in H^{1}(K)^{2} .
$$

5.2.2. The Durán and Liberman element. This modification of the MITC3 FEM adopts all notations $W_{h}, \boldsymbol{\Gamma}_{h}, \boldsymbol{R}_{h}$ from the previous subsection, therefore (5.1) holds. The rotation space reads

$$
\boldsymbol{\Theta}_{h}:=\left(S_{0}^{1}\left(\mathcal{T}_{h}\right)\right)^{2} \oplus B_{h} .
$$

Therein, the edge bubble function space $B_{h}$ is defined as follows. Given an element $K \in \mathcal{T}_{h}$, its barycentric coordinates and unit tangential vectors are denoted by $\left\{\lambda_{i}\right\}_{i=1}^{3}$ and $\left\{\boldsymbol{\tau}_{i}\right\}_{i=1}^{3}$, respectively. Define

$$
\begin{gathered}
\boldsymbol{\psi}_{1}:=\lambda_{2} \lambda_{3} \boldsymbol{\tau}_{1}, \boldsymbol{\psi}_{2}:=\lambda_{3} \lambda_{1} \boldsymbol{\tau}_{2}, \boldsymbol{\psi}_{3}:=\lambda_{1} \lambda_{2} \boldsymbol{\tau}_{3} \\
B_{h}:=\left\{\boldsymbol{\beta} \in H_{0}^{1}(\Omega)^{2}:\left.\boldsymbol{\beta}\right|_{K} \in \operatorname{span}\left\{\boldsymbol{\psi}_{1}, \boldsymbol{\psi}_{2}, \boldsymbol{\psi}_{3}\right\}, K \in \mathcal{T}_{h}\right\} .
\end{gathered}
$$

The Durán and Liberman FEM is a locking free element for the Reissner-Mindlin plate problem with robust a priori error estimate established in [26].

Since the proof of Subsection 5.2.1 also covers this case, (5.1) holds for this DL element.

5.2.3. High-order triangle MITC elements. This subsection presents three families of high-order triangle MITC elements. These elements are proposed and analyzed in [18. The lowest element order among them is the usual MITC7 element which appeared in [11].

Family $I$. We define for $k \geq 2$,

$$
\begin{gathered}
W_{h}:=\left\{v \in H_{0}^{1}(\Omega):\left.v\right|_{K} \in Q_{k}(K), K \in \mathcal{T}_{h}\right\}, \\
\boldsymbol{\Theta}_{h}:=\left\{\boldsymbol{\beta} \in H_{0}^{1}(\Omega)^{2}:\left.\boldsymbol{\beta}\right|_{K} \in \boldsymbol{\Theta}_{k}(K), K \in \mathcal{T}_{h}\right\},
\end{gathered}
$$

where

$$
\boldsymbol{\Theta}_{k}(K):= \begin{cases}\left(Q_{k}(K)\right)^{2} & \text { for } k \geq 4 \\ \left(S_{k}(K)\right)^{2} & \text { for } k=2,3\end{cases}
$$

and

$$
S_{k}(K):=\left\{w \in Q_{k+1}(K):\left.w\right|_{E} \in Q_{k}(E) \text { for every edge } E \text { of } K\right\} .
$$

For the shear force space $\boldsymbol{\Gamma}_{h}$ we take the rotated Raviart-Thomas space

$$
\boldsymbol{\Gamma}_{h}:=\left\{\boldsymbol{\sigma} \in H_{0}(\operatorname{rot}, \Omega): \boldsymbol{\sigma} \in\left(Q_{k-1}(K)\right)^{2} \oplus Q_{k-1}(K)(y,-x)^{T}, K \in \mathcal{T}_{h}\right\},
$$


with the reduction operator $\boldsymbol{R}_{h}$ defined through

$$
\begin{gathered}
\int_{E}\left(\boldsymbol{R}_{h} \boldsymbol{\sigma}-\boldsymbol{\sigma}\right) \cdot \boldsymbol{\tau}_{E} v d s=0, v \in Q_{k-1}(E) \text { for every edge } E \text { of every } K \in \mathcal{T}_{h}, \\
\int_{K}\left(\boldsymbol{R}_{h} \boldsymbol{\sigma}-\boldsymbol{\sigma}\right) \cdot \boldsymbol{\beta} d x d y=0 \text { for every } \boldsymbol{\beta} \in\left(Q_{k-2}(K)\right)^{2} .
\end{gathered}
$$

Family II. Let $S_{k}$ be as in Family I, and define

$$
W_{h}:=\left\{v \in H_{0}^{1}(\Omega):\left.v\right|_{K} \in S_{k}(K), K \in \mathcal{T}_{h}\right\} .
$$

We choose the BDFM space

$$
\begin{aligned}
\boldsymbol{\Gamma}_{h}:=\left\{\boldsymbol{\sigma} \in H_{0}(\operatorname{rot}, \Omega):\left.\boldsymbol{\sigma}\right|_{K} \in\left(Q_{k}(K)\right)^{2}, \boldsymbol{\sigma} \cdot \boldsymbol{\tau}_{E} \in Q_{k-1}(E)\right. & \\
& \text { for every edge } \left.E \text { of } K \in \mathcal{T}_{h}\right\}
\end{aligned}
$$

as the shear force space $\boldsymbol{\Gamma}_{h}$ with the reduction operator defined as

$$
\begin{gathered}
\int_{E}\left(\boldsymbol{R}_{h} \boldsymbol{\sigma}-\boldsymbol{\sigma}\right) \cdot \boldsymbol{\tau}_{E} v d s=0, v \in Q_{k-1}(E) \text { for every edge } E \text { of } K, \\
\int_{K}\left(\boldsymbol{R}_{h} \boldsymbol{\sigma}-\boldsymbol{\sigma}\right) \cdot \boldsymbol{\beta} d x=0 \text { for every } \boldsymbol{\beta} \in\left(Q_{k-2}(K)\right)^{2}, \\
\int_{K}\left(\boldsymbol{R}_{h} \boldsymbol{\sigma}-\boldsymbol{\sigma}\right) \cdot \nabla \psi_{j} d x=0 \text { for every } j=0, \cdots, k-2,
\end{gathered}
$$

where $\psi_{0}, \cdots, \psi_{k-2}$ are arbitrary polynomials in $Q_{k}(K)$, chosen once and for all, with $\triangle \psi_{j}=x^{j} y^{k-j-2}, j=0, \cdots, k-2$. The rotation space is the same as in Family I.

Family III. In this case let

$$
W_{h}:=\left\{v \in H_{0}^{1}(\Omega):\left.v\right|_{K} \in Q_{k+1}(K), K \in \mathcal{T}_{h}\right\}
$$

and use the BDM space

$$
\boldsymbol{\Gamma}_{h}:=\left\{\boldsymbol{\sigma} \in H_{0}(\operatorname{rot}, \Omega):\left.\boldsymbol{\sigma}\right|_{K} \in\left(Q_{k}(K)\right)^{2}, K \in \mathcal{T}_{h}\right\} .
$$

The reduction operator $\boldsymbol{R}_{h}$ is defined through

$$
\begin{gathered}
\int_{E}\left(\boldsymbol{R}_{h} \boldsymbol{\sigma}-\boldsymbol{\sigma}\right) \cdot \boldsymbol{\tau}_{E} v d s=0, v \in Q_{k}(E) \text { for every edge } E \text { of } K, \\
\int_{K}\left(\boldsymbol{R}_{h} \boldsymbol{\sigma}-\boldsymbol{\sigma}\right) \cdot \boldsymbol{\beta} d x d y=0 \text { for every } \boldsymbol{\beta} \in\left(Q_{k-2}(K)\right)^{2}, \\
\int_{K}\left(\boldsymbol{R}_{h} \boldsymbol{\sigma}-\boldsymbol{\sigma}\right) \cdot \nabla \psi_{j} d x d y=0 \text { for every } j=0, \cdots, k-2,
\end{gathered}
$$

with $\psi_{j}$ and the rotation space $\boldsymbol{\Theta}_{h}$ from Family II. Since $\left(S_{0}^{1}\left(\mathcal{T}_{h}\right)\right)^{2} \subset \boldsymbol{\Gamma}_{h}$ when $k \geq 2$, it holds for all of those elements that

$$
\mu_{h}\left(\gamma_{h}\right)=0 \text {. }
$$

5.3. Quadrilateral MITC elements. This subsection discusses quadrilateral MITC elements. 
5.3.1. The rectangular MITC4 element. In this case, we restrict ourselves to the rectangular mesh, and define

$W_{h}:=\left\{v \in H_{0}^{1}(\Omega):\left.v\right|_{K} \in Q_{1}(K), K \in \mathcal{T}_{h}\right\}$,

$\boldsymbol{\Theta}_{h}:=\left\{\boldsymbol{\beta} \in H_{0}^{1}(\Omega)^{2}:\left.\boldsymbol{\beta}\right|_{K} \in\left(Q_{1}(K)\right)^{2}, K \in \mathcal{T}_{h}\right\}$,

$\boldsymbol{\Gamma}_{h}:=\left\{\boldsymbol{\sigma} \in H_{0}(\operatorname{rot}, \Omega):\left.\boldsymbol{\sigma}\right|_{K} \in\left(Q_{0}(K)\right)^{2} \oplus Q_{0}(K)(y, 0)^{T} \oplus Q_{0}(K)(0, x)^{T}, K \in \mathcal{T}_{h}\right\}$,

with the reduction operator $\boldsymbol{R}_{h}$ defined as, for any $K \in \mathcal{T}_{h}$,

$$
\int_{E}\left(\boldsymbol{R}_{h} \boldsymbol{\sigma}-\boldsymbol{\sigma}\right) \cdot \boldsymbol{\tau}_{E} d s=0 \text { for every edge } E \text { of } K \text {. }
$$

This element is locking free under the condition that the mesh $\mathcal{T}_{h}$ is obtained from a coarser mesh $\mathcal{T}_{2 h}$ through bisectioning [27, 29]. It is well-known that

$$
\left(\boldsymbol{\sigma}_{h}, \boldsymbol{R}_{h} \boldsymbol{\psi}_{h}-\boldsymbol{\psi}_{h}\right)_{L^{2}(\Omega)}=0 \text { for any } \boldsymbol{\sigma}_{h} \in \boldsymbol{\Gamma}_{h} \text { and } \boldsymbol{\psi}_{h} \in\left(S_{0}^{1}\left(\mathcal{T}_{h}\right)\right)^{2} .
$$

Therefore

$$
\mu_{h}\left(\gamma_{h}\right)=0
$$

5.3.2. The rectangular DHHLR element. This is a modification of the MITC4 element with

$$
\Theta_{h}:=\left(S_{0}^{1}\left(\mathcal{T}_{h}\right)\right)^{2} \oplus B_{h} .
$$

The edge bubble function space $B_{h}$ is defined as follows. For each edge $\hat{E}_{i}$ of $\hat{K}$, $i=1,2,3,4$, let $\hat{\psi}_{i}$ denote the bicubic functions vanishing on $\hat{E}_{j}$ when $j \neq i$. Then we define $\boldsymbol{\psi}_{i}=\hat{\boldsymbol{\psi}}_{i} \circ F_{K}^{-1} \boldsymbol{\tau}_{E_{i}}$ and set

$$
B_{h}:=\left\{\boldsymbol{\beta} \in H_{0}^{1}(\Omega)^{2}:\left.\boldsymbol{\beta}\right|_{K} \in \operatorname{span}\left\{\boldsymbol{\psi}_{1}, \boldsymbol{\psi}_{2}, \boldsymbol{\psi}_{3}, \boldsymbol{\psi}_{4}\right\}, K \in \mathcal{T}_{h}\right\} .
$$

With the displacement space $W_{h}$, the shear force space $\boldsymbol{\Gamma}_{h}$ and the reduction operator $\boldsymbol{R}_{h}$ from the MITC4 element, this is a locking free element for the ReissnerMindlin plate problem. Robust a priori error estimate was obtained in [27. Also, (5.3) holds.

Remark 5.3. Another variant for the MITC4 element is the Hu element 29]. Instead of the rotated Raviart-Thomas element, the quadrilateral ABF element from 6 is employed to approximate the shear force in this element and four interior bubble functions yield stability. Compared to other lowest quadrilateral elements, the convergence of this method is independent of the mesh distortion parameter $\alpha$ defined in Subsection 5.3.3; cf. 29] for further details. For this element, one can also prove $\mu_{h}\left(\gamma_{h}\right)=0$.

5.3.3. High-order quadrilateral MITC elements. We first introduce some notations. The distance between the midpoints of two diagonals of $K$ is denoted by $d_{K}$ and $\mathcal{T}_{h}$ is supposed to satisfy the $(1+\alpha)$-section condition [39], i.e., $d_{K}$ is of order $\mathcal{O}\left(h_{K}^{1+\alpha}\right)$ uniformly for all elements $K$ as $h$ tends to zero for $0 \leq \alpha \leq 1$; the 2-section condition is also called bi-section condition [42].

Given any quadrilateral $K \in \mathcal{T}_{h}$ with four nodes $p_{j}\left(x_{j}, y_{j}\right), j=1, \cdots, 4$ numbered counterclockwise, let $\hat{p}_{j}\left(\xi_{j}, \eta_{j}\right), j=1, \cdots, 4$ be the nodes of $\hat{K}$. Then the bilinear transformation $F_{K}$ takes the form

$$
x=\sum_{j=1}^{4} x_{j} N_{j}(\xi, \eta), \quad y=\sum_{j=1}^{4} y_{j} N_{j}(\xi, \eta), \quad(\xi, \eta) \in \hat{K},
$$


with the bilinear basis functions $N_{j}(\xi, \eta)$ defined by

$$
\begin{array}{ll}
N_{1}(\xi, \eta)=\frac{1}{4}(1-\xi)(1-\eta), & N_{2}(\xi, \eta)=\frac{1}{4}(1+\xi)(1-\eta), \\
N_{3}(\xi, \eta)=\frac{1}{4}(1+\xi)(1+\eta), & N_{4}(\xi, \eta)=\frac{1}{4}(1-\xi)(1+\eta) .
\end{array}
$$

Define some coefficients $c_{0}, c_{1}, c_{2}, c_{12}, d_{0}, d_{1}, d_{2}, d_{12}$ through

$$
\left(\begin{array}{cc}
c_{0} & d_{0} \\
c_{1} & d_{1} \\
c_{2} & d_{2} \\
c_{12} & d_{12}
\end{array}\right)=\frac{1}{4}\left(\begin{array}{cccc}
1 & 1 & 1 & 1 \\
-1 & 1 & 1 & -1 \\
-1 & -1 & 1 & 1 \\
1 & -1 & 1 & -1
\end{array}\right)\left(\begin{array}{ll}
x_{1} & y_{1} \\
x_{2} & y_{2} \\
x_{3} & y_{3} \\
x_{4} & y_{4}
\end{array}\right)
$$

Then the Jacobian matrix of $F_{K}$ reads

$$
D F_{K}=\left(\begin{array}{ll}
\partial x / \partial \xi & \partial x / \partial \eta \\
\partial y / \partial \xi & \partial y / \partial \eta
\end{array}\right)=\left(\begin{array}{cc}
c_{1}+c_{12} \eta & c_{2}+c_{12} \xi \\
d_{1}+d_{12} \eta & d_{2}+d_{12} \xi
\end{array}\right)
$$

with the determinant $J_{K}(\xi, \eta)=J_{0}+J_{1} \xi+J_{2} \eta$ and its inverse

$$
D F_{K}^{-1}=\left(\begin{array}{ll}
\partial \xi / \partial x & \partial \xi / \partial y \\
\partial \eta / \partial x & \partial \eta / \partial y
\end{array}\right)=\frac{1}{J_{K}(\xi, \eta)}\left(\begin{array}{cc}
d_{2}+d_{12} \xi & -c_{2}-c_{12} \xi \\
-d_{1}-d_{12} \eta & c_{1}+c_{12} \eta
\end{array}\right) .
$$

Here and throughout this paper $J_{0}=c_{1} d_{2}-c_{2} d_{1}, J_{1}=c_{1} d_{12}-c_{12} d_{1}$, and $J_{2}=$ $c_{12} d_{2}-c_{2} d_{12}$. In terms of the aforementioned mesh parameters, $d_{K}=\mathcal{O}\left(h_{K}^{1+\alpha}\right)$ implies

$$
\left|\hat{\nabla} J_{K}\right|+h_{K}^{-1}\left|J_{1}\right|+h_{K}^{-1}\left|J_{2}\right|+\left|c_{12}\right|+\left|d_{12}\right| \lesssim h_{K}^{1+\alpha} .
$$

For $S \subset R^{2}$, we let $P_{k}(S)$ denote the set of polynomials of total degree $\leq k$, and $Q_{k}^{\prime}(S)$ denote the "trunk" or "serendipity" space of polynomials. The spaces $W_{h}$ and $\boldsymbol{\Theta}_{h}$ are defined as

$$
\begin{gathered}
W_{h}:=\left\{v \in H_{0}^{1}(\Omega):\left.v\right|_{K}=\hat{v} \circ F_{K}^{-1}, \hat{v} \in W_{k}(\hat{K}), K \in \mathcal{T}_{h}\right\}, \\
\boldsymbol{\Theta}_{h}:=\left\{\boldsymbol{\beta} \in\left(H_{0}^{1}(\Omega)\right)^{2}:\left.\boldsymbol{\beta}\right|_{K}=\hat{\boldsymbol{\beta}} \circ F_{K}^{-1}, \hat{\boldsymbol{\beta}} \in \boldsymbol{\Theta}_{k}(\hat{K}), K \in \mathcal{T}_{h}\right\},
\end{gathered}
$$

where $W_{k}(\hat{K})$ and $\boldsymbol{\Theta}_{k}(\hat{K})$, which will be specified in the sequel, are polynomial spaces on the reference element $\hat{K}$. The space $\boldsymbol{\Gamma}_{h}$ is defined differently by

$$
\boldsymbol{\Gamma}_{h}:=\left\{\boldsymbol{\sigma} \in H_{0}(\operatorname{rot}, \Omega):\left.\boldsymbol{\sigma}\right|_{K} \in \boldsymbol{\Gamma}_{k}(K), K \in \mathcal{T}_{h}\right\}
$$

for the space $\boldsymbol{\Gamma}_{k}(K)$ defined from the space $\boldsymbol{\Gamma}_{k}(\hat{K})$ on the reference square through the following Piola transformation for the operator rot,

$$
\boldsymbol{\Gamma}_{k}(K):=\left\{\boldsymbol{\sigma}: \boldsymbol{\sigma}=D F_{K}^{-T} \overline{\boldsymbol{\sigma}} \circ F_{K}^{-1}, \overline{\boldsymbol{\sigma}} \in \boldsymbol{\Gamma}_{k}(\hat{K})\right\} .
$$

The reduction operator $\boldsymbol{R}_{h}$ is also defined locally on each element from the reduction operator $\widehat{\boldsymbol{R}}_{\hat{K}}$ defined on the reference element with the same transformation:

$$
\left.\boldsymbol{R}_{h} \boldsymbol{\sigma}\right|_{K}=D F_{K}^{-T} \widehat{\boldsymbol{R}}_{\hat{K}} \overline{\boldsymbol{\sigma}} \circ F_{K}^{-1}, \text { with } \overline{\boldsymbol{\sigma}}=D F_{K}^{T} \hat{\boldsymbol{\sigma}}=D F_{K}^{T} \boldsymbol{\sigma} \circ F_{K} .
$$

We consider four families of quadrilateral MITC elements for the ReissnerMindlin plate problem. The rectangular version of these elements is proposed and analyzed in [43. We refer to [29, 30] for the a priori error analysis of the general case. 
Family I. In this family, the displacement and the rotation spaces read, respectively,

$$
\begin{gathered}
W_{k}(\hat{K}):=Q_{\hat{K}} \cap P_{k+1}(\hat{K}) . \\
\Theta_{k}(\hat{K}):=\left[Q_{k}(\hat{K}) \cap P_{k+2}(\hat{K})\right]^{2} .
\end{gathered}
$$

For $\boldsymbol{\Gamma}_{h}$, we choose $\boldsymbol{\Gamma}_{k}(\hat{K})$ as the following BDFM space [17]:

$$
\boldsymbol{\Gamma}_{k}(\hat{K}):=\left\{\overline{\boldsymbol{\sigma}} \mid \overline{\boldsymbol{\sigma}} \in\left[P_{k}(\hat{K}) \backslash\left\{\hat{x}^{k}\right\} \times P_{k}(\hat{K}) \backslash\left\{\hat{y}^{k}\right]\right\}\right\} .
$$

The reduction operator $\widehat{\boldsymbol{R}}_{\hat{K}}$ is defined by

$$
\begin{gathered}
\int_{\hat{E}}\left[\left(\widehat{\boldsymbol{R}}_{\hat{K}} \overline{\boldsymbol{\sigma}}-\overline{\boldsymbol{\sigma}}\right) \cdot \hat{\boldsymbol{\tau}}\right] \hat{w} d \hat{s}=0 \quad \text { for all } \hat{w} \in P_{k-1}(\hat{E}) \quad \text { and for every edge } \hat{E} \text { of } \hat{K}, \\
\int_{\hat{K}}\left(\widehat{\boldsymbol{R}}_{\hat{K}} \overline{\boldsymbol{\sigma}}-\overline{\boldsymbol{\sigma}}\right) \cdot \hat{\boldsymbol{v}} d \hat{x} d \hat{y}=0, \quad \forall \hat{\boldsymbol{v}} \in\left[P_{k-2}(\hat{K})\right]^{2}
\end{gathered}
$$

Theorem 5.4. With $\alpha=1$, it holds that

$$
\mu_{h}\left(\gamma_{h}\right)^{2} \lesssim \sum_{K \in \mathcal{T}_{h}} h_{K}^{2}\left(h_{K}^{2}+t^{2}\right)\left\|\operatorname{div} \gamma_{h}+g\right\|_{L^{2}(K)}^{2}+\sum_{K \in \mathcal{T}_{h}} h_{K}^{2(k+1)}\|g\|_{L^{2}(K)}^{2}
$$

Remark 5.5. Since the a priori convergence rate is of order $k$ in the energy norm, the second term on the right-hand side of (5.6) is always a high-order term provided that $g \in L^{2}(\Omega)$.

Proof. For $k \geq 3$, we have

$$
D F_{K}^{T} \hat{\boldsymbol{\beta}} \in \boldsymbol{\Gamma}_{k}(\hat{K}) \text { for every } \hat{\boldsymbol{\beta}} \in\left(Q_{1}(\hat{K})\right)^{2},
$$

which implies

$$
\mu_{h}\left(\gamma_{h}\right)=0 .
$$

It remains to prove (5.6) for the case $k=2$. By the definitions of $\gamma_{h}$ and $\boldsymbol{R}_{h}$,

$$
\boldsymbol{R}_{h} \boldsymbol{\beta}-\left.\boldsymbol{\beta}\right|_{K}=\left.M^{-1}\left(\widehat{\boldsymbol{R}}_{\hat{K}} M \widehat{\boldsymbol{\beta}}-M \widehat{\boldsymbol{\beta}}\right)\right|_{K},
$$

with $M=D F_{K}^{T}$ and $\widehat{\boldsymbol{\beta}}=\left(a_{0}, b_{0}\right)^{T}+\left(a_{1}, b_{1}\right) \hat{x}+\left(a_{2}, b_{2}\right)^{T} \hat{y}+\left(a_{3}, b_{3}\right)^{T} \hat{x} \hat{y}$. The definitions of $\boldsymbol{\Gamma}_{k}(\hat{K})$ with $k=2$ and $D F_{K}^{T}$ lead to

$$
\widehat{\boldsymbol{R}}_{\hat{K}} M \widehat{\boldsymbol{\beta}}-M \widehat{\boldsymbol{\beta}}=\left(a_{3} c_{12}+b_{3} d_{12}\right)\left(\hat{x}-\hat{x} \hat{y}^{2}, \hat{y}-\hat{x}^{2} \hat{y}\right)^{T}
$$

with $c_{12}$ and $d_{12}$ from (5.4). Define

$$
\widehat{\psi}=\frac{a_{3} c_{12}+b_{3} d_{12}}{2}\left(-1+\hat{x}^{2}+\hat{y}^{2}-\hat{x}^{2} \hat{y}^{2}\right) .
$$

Then it holds that

$$
\widehat{\nabla} \widehat{\psi}=\widehat{\boldsymbol{R}}_{\hat{K}} M \widehat{\boldsymbol{\beta}}-M \widehat{\boldsymbol{\beta}} .
$$

It follows from the mesh parameter estimate (5.5) that

$$
\|\widehat{\nabla} \widehat{\psi}\|_{L^{2}(\hat{K})} \lesssim h_{K}^{1+\alpha}\|\widehat{\nabla} \widehat{\boldsymbol{\beta}}\|_{L^{2}(\hat{K})} .
$$

Let $\psi_{K}$ be defined through the following relation:

$$
\psi_{K}:=\widehat{\psi} \circ F_{K}^{-1} .
$$


Note that $\psi_{K} \in H_{0}^{1}(K)$ and $\nabla \psi=M^{-1} \widehat{\nabla} \widehat{\psi}$. With $\psi=\sum_{K \in \mathcal{T}_{h}} \psi_{K} \in H_{0}^{1}(\Omega)$, the

Poincaré inequality and (5.7) elementwise, one proves

$$
\begin{aligned}
\left(\gamma_{h},\right. & \left.\boldsymbol{R}_{h} \boldsymbol{\beta}-\boldsymbol{\beta}\right)_{L^{2}(\Omega)}=\sum_{K \in \mathcal{T}_{h}}\left(\gamma_{h}, M^{-1}\left(\widehat{\boldsymbol{R}}_{\hat{K}} M \widehat{\boldsymbol{\beta}}-M \widehat{\boldsymbol{\beta}}\right)\right)_{L^{2}(K)} \\
& =\left(\gamma_{h}, \nabla \psi\right)_{L^{2}(\Omega)}=\sum_{K \in \mathcal{T}_{h}}\left(\operatorname{div} \gamma_{h}+g, \psi_{K}\right)_{L^{2}(K)}-\sum_{K \in \mathcal{T}_{h}}\left(g, \psi_{K}\right)_{L^{2}(K)} \\
& \lesssim \sum_{K \in \mathcal{T}_{h}} h_{K}\left\|\operatorname{div} \gamma_{h}+g\right\|_{L^{2}(K)}\left\|\nabla \psi_{K}\right\|_{L^{2}(K)}+h_{K}\|g\|_{L^{2}(K)}\left\|\nabla \psi_{K}\right\|_{L^{2}(K)} \\
& \lesssim \sum_{K \in \mathcal{T}_{h}} h_{K}^{2+\alpha}\left\|\operatorname{div} \gamma_{h}+g\right\|_{L^{2}(K)}\|\boldsymbol{\beta}\|_{H^{1}(K)}+h_{K}^{2+\alpha}\|g\|_{L^{2}(K)}\|\boldsymbol{\beta}\|_{H^{1}(K)}
\end{aligned}
$$

This implies

$$
\begin{aligned}
\mu_{h}\left(\gamma_{h}\right) \lesssim & \left(\sum_{K \in \mathcal{T}_{h}} h_{K}^{2}\left(h_{K}^{2}+t^{2}\right)\left\|\operatorname{div} \gamma_{h}+g\right\|_{L^{2}(K)}^{2}\right)^{1 / 2} \\
& +\left(\sum_{K \in \mathcal{T}_{h}} h_{K}^{6}\|g\|_{L^{2}(K)}^{2}\right)^{1 / 2} \cdot \square
\end{aligned}
$$

Family II. In this method, $W_{h}$ and $\boldsymbol{\Gamma}_{h}$ are the same as in Family I with a different choice of the rotation space, which reads as

$$
\Theta_{k}(\hat{K})=\left[Q_{k}(\hat{K})\right]^{2} .
$$

For these elements, the estimate (5.6) holds equally.

Family III. The spaces for the rotation are chosen as in Family I. However, we take the following BDM space [17,

$$
\boldsymbol{\Gamma}_{k}(\hat{K})=\left\{\overline{\boldsymbol{\sigma}} \mid \overline{\boldsymbol{\sigma}} \in\left[P_{k}(\hat{K})\right]^{2} \oplus \hat{\nabla}\left(\hat{x} \hat{y}^{k+1}\right) \oplus \widehat{\nabla}\left(\hat{x}^{k+1} \hat{y}\right)\right\},
$$

as the shear force space with the reduction operator defined by

$$
\begin{gathered}
\int_{\hat{E}}\left[\left(\widehat{\boldsymbol{R}}_{\hat{K}} \overline{\boldsymbol{\sigma}}-\overline{\boldsymbol{\sigma}}\right) \cdot \hat{\boldsymbol{\tau}}\right] \hat{w} d \hat{s}=0, \quad \forall \hat{w} \in P_{k}(\hat{E}) \text { for every edge } \hat{E} \text { of } \hat{K}, \\
\int_{\hat{K}}\left(\widehat{\boldsymbol{R}}_{\hat{K}} \overline{\boldsymbol{\sigma}}-\overline{\boldsymbol{\sigma}}\right) \cdot \hat{\boldsymbol{v}} d \hat{x} d \hat{y}=0, \quad \forall \hat{\boldsymbol{v}} \in\left[P_{k-2}(\hat{K})\right]^{2} .
\end{gathered}
$$

Therefore, the deflection space has to be selected as

$$
W_{k}(\hat{K})=Q_{k+1}^{\prime}(\hat{K}) \text {. }
$$

Theorem 5.6. With $\alpha=1$, it holds that

$$
\mu_{h}\left(\gamma_{h}\right)^{2} \lesssim \sum_{K \in \mathcal{T}_{h}} h_{K}^{2}\left(h_{K}^{2}+t^{2}\right)\left\|\operatorname{div} \gamma_{h}+g\right\|_{L^{2}(K)}^{2}+\sum_{K \in \mathcal{T}_{h}} h_{K}^{2(k+1)}\|g\|_{L^{2}(K)}^{2}
$$

Proof. Arguing in the same way as for (5.6) shows the asserted result; the details are omitted.

Family IV. The rotation space reads

$$
\boldsymbol{\Theta}_{k}(\hat{K})=\left\{\boldsymbol{\psi} \in\left[Q_{k+1}(\hat{K})\right]^{2}|\boldsymbol{\psi}|_{\hat{E}} \in\left[P_{k}(\hat{K})\right]^{2} \text { for every edge } \hat{E} \text { of } \hat{K}\right\} .
$$

The corresponding space for the shear is the following rotated Raviart-Thomas space over quadrilaterals,

$$
\boldsymbol{\Gamma}_{k}(\hat{K})=\left\{\overline{\boldsymbol{\sigma}} \mid \overline{\boldsymbol{\sigma}} \in Q_{k-1, k}(\hat{K}) \times Q_{k, k-1}(\hat{K})\right\},
$$


with the reduction operator defined by

$$
\begin{gathered}
\int_{\hat{E}}\left[\left(\widehat{\boldsymbol{R}}_{\hat{K}} \overline{\boldsymbol{\sigma}}-\overline{\boldsymbol{\sigma}}\right) \cdot \hat{\boldsymbol{\tau}}\right] \hat{w} d \hat{s}=0, \quad \forall \hat{w} \in P_{k-1}(\hat{E}) \text { for every edge } \hat{E} \text { of } \hat{K}, \\
\int_{\hat{K}}\left(\widehat{\boldsymbol{R}}_{\hat{K}} \overline{\boldsymbol{\sigma}}-\overline{\boldsymbol{\sigma}}\right) \cdot \hat{\boldsymbol{v}} d \hat{x} d \hat{y}=0, \quad \forall \hat{\boldsymbol{v}} \in Q_{k-1, k-2}(\hat{K}) \times Q_{k-2, k-1}(\hat{K}) .
\end{gathered}
$$

The space for the deflection is selected as

$$
W_{k}(\hat{K})=Q_{k}(\hat{K}) \text {. }
$$

Since

$$
D F_{K}^{T} \hat{\boldsymbol{\beta}} \in \boldsymbol{\Gamma}_{k}(\hat{K}) \text { for every } \hat{\boldsymbol{\beta}} \in\left(Q_{1}(\hat{K})\right)^{2}
$$

for $k \geq 2$, it holds that $\mu_{h}\left(\gamma_{h}\right)=0$.

\section{ACKNOWLEDGMENTS}

The authors would like to thank one referee for pointing out a small gap in the proof of (3.6) in the previous version of this paper, which lead to the revision of Remark 3.1. The second author JH thankfully acknowledges the Alexander von Humboldt Fellowship during his stay at the Department of Mathematics at Humboldt-Universität zu Berlin, Germany.

\section{REFERENCES}

[1] M. Ainsworth and J.T. Oden, A posteriori error estimation in finite element analysis, Wiley-Interscience [John Wiley \& Sons], New York, 2000. MR1885308 (2003b:65001)

[2] D.N. Arnold and F. Brezzi, Mixed and nonconforming finite element methods: implementation, postprocessing and error estimates, $\mathrm{M}^{2} \mathrm{AN}, 19(1985)$, pp. 7-32. MR813687(87g:65126)

[3] D.N. Arnold and F. Brezzi, Some new elements for the Reissner-Mindlin plate model, J.L.Lions, C.Baiocchi(eds.), Boundary value problem for partial differential equations and applications, Masson, 1993, pp. 287-292. MR1260452 (94k:73066)

[4] D.N. Arnold and R.S. Falk, A uniformly accurate finite element method for Reissner-Mindlin plates, SIAM. J.Numer. Anal., 26(1989), pp. 1276-1290. MR.1025088(91c:65068)

[5] D.N. Arnold and R.S. Falk, The boundary layer for the Reissner-Mindlin plate model, SIAM J.Math.Anal. 21(1990), pp. 281-312. MR.1038893 (91c:73053)

[6] D.N. Arnold, D. Boffi and R.S. Falk, Quadrilateral H(div) finite elements, SIAM J. Numer. Anal. 42 (2005), pp. 2429-2451. MR2139400 (2006d:65129)

[7] I. Babuska, A. Miller, A feedback finite element method with a posteriori error estimation: Part I. The finite element method and some basic properties of the a posteriori error estimator, Comp. Meth. Appl. Mech. Engrg., 61 (1987), pp. 1-40 MR880421 (88d:73036)

[8] I. Babuška and W.C. Rheinboldt, A posteriori error analysis of finite element solutions for one-dimensional problems, SIAM J.Numer. Anal., 18 (1981), pp. 565-589. MR615532 (82j:65082)

[9] I. Babuška and T. Strouboulis, The Finite Element Method and its Reliability, The Clarendon Press Oxford University Press, 2001. MR.1857191 (2002k:65001)

[10] K. J. Bathe, F. Brezzi and M. Fortin, A simplified analysis of two-plate elements: The MITC4 and MITC9 element, G.N. Pande and J. Middleton (eds), Numeta 87 Vol. 1, Numerical Techniques for Engineering Analysis and Design,Martinus Nijhoff, Amsterdam.

[11] K. J. Bathe, F. Brezzi and M. Fortin, Mixed-interpolated elements for Reissner-Mindlin plates, Int. J. Num. Meths. Engrg., 28(1989), pp. 1787-1801. MR.1008138 (90g:73090)

[12] K. J. Bathe and E. Dvorkin, A four-node plate bending element based on Mindlin-Reissner plate theory and a mixed interpolation, Int. J. Num. Meths. Engrg., 21(1985), pp. 367-383.

[13] D. Braess, and P. Peisker, Uniform convergence of mixed interpolated elements for ReissnerMindlin plates, $\mathrm{M}^{2}$ AN., 26(1992), pp. 557-574. MR1177387 (93j:73070)

[14] D. Braess, Finite Elements, Cambridge University Press, 1997. MR1463151 (98f:65002)

[15] S.C. Brenner, L.R. Scott, The Mathematical Theory of Finite Element Methods, Springer Verlag, Second Edition, 2002. MR1894376 (2003a:65103) 
[16] F. Brezzi and M. Fortin, Numerical approximation of Reissner-Mindlin plates, Math Comp., 47(1986), pp. 151-158. MR842127 (87g:73057)

[17] F. Brezzi, M. Fortin, Mixed and Hybrid Finite Element Methods, Springer, Berlin, 1991. MR:1115205 (92d:65187)

[18] F. Brezzi, M. Fortin and R. Stenberg, Error analysis of mixed-interpolated elements for Reissner-Mindlin plate, Math. Models. Meth. Appl. Sci., 1 (1991), pp. 125-151. MR1115287 (92e:73030)

[19] C. Bernardi, V. Girault, A local regularisation operator for triangular and quadrilateral finite elements, SIAM J. Numer. Anal. 35 (1998), pp. 1893-1916. MR1639966 (99g:65107)

[20] C. Carstensen, Quasi-interpolation and a posteriori error analysis in finite element methods, Math. Model. Numer. Anal., 33 (1999), 1187-1202. MR.1736895(2001a:65135)

[21] Carsten Carstensen, Residual-Based a posteriori error estimate for a nonconforming Reissner-Mindlin plate finite element, SIAM J. Numer. Anal., 39(2002), pp. 2034-2044. MR 1897948 (2003e:65213)

[22] C. Carstensen and J. Schöberl, Residual-Based a posteriori error estimate for a mixed Reissner-Mindlin plate finite element, Numer.Math., 103 (2006), pp. 225-250. MR 2222809 (2007c:74055)

[23] D. Chapelle and R. Stenberg, An optimal low-order locking-free finite element method for Reissner-Mindlin plates, Math. Model Meth. Appl. Sci. 8 (1998), pp. 407-430. MR1624871 (99d:73088)

[24] P.G. Ciarlet, The Finite Element Method for Elliptic Problems. North-Holland, 1978; reprinted as SIAM Classics in Applied Mathematics, 2002. MR0520174 (58:25001)

[25] P. Clément, Approximation by finite element functions using local regularization, RAIRO Anal. Numér., 9 (1975), pp. 77-84. MR0400739 (53:4569)

[26] R. Durán and E. Liberman, On mixed finite element methods for Reissner-Mindlin plate model, Math. Comp., 58 (1992), pp. 561-573. MR 1106965 (92f:65135)

[27] R. Durán, E. Hernández, L. Hervella-Nieto, E. Liberman, and R. Rodríguez, Error estimates for lower-order isoparametric quadrilateral finite elements for plates, SIAM. J. Numer. Anal., 41 (2003), pp. 1751-1772. MR2035005 (2004m:65192)

[28] I. Fried and S.K. Yang, Triangular, nine-degrees-of-freedoms, plate bending element of quadratic accuracy, Quart. Appl. Math., 31 (1973), pp. 303-312.

[29] J. Hu, Quadrilateral locking free elements in elasticity, Doctorate Dissertation (in Chinese), Institute of Computational Mathematics, Chinese Academy of Science (2004).

[30] J. Hu and Z.C. Shi, Analysis for quadrilateral MITC elements for Reissner-Mindlin plate, Preprint 2003-12, Institute of Computational Mathematics, Chinese Academy of Sciences, www.cc.ac.cn/quadri_mitc.ps, Submitted to Math. Comp., 2005.

[31] T.J.R. Hughes, R.L. Taylor and W. Kanoknukuchai, A simple and efficient element for plate bending, Int. J. Numer. Meth. Engrg., 11 (1977), pp. 1529-1543.

[32] T.J.R. Hughes, M. Cohen and M. Haroun, Reduced and selective integration techniques in the finite element analysis of plates, Nuclear Engineering and Design, 46 (1978), pp. 203-222.

[33] T.J.R. Hughes, The finite element method: Linear static and dynamic finite element analysis, Prentice-Hall, Englewood Cliffs, New Jersey, 1987. MR.1008473 (90i:65001)

[34] T.J.R. Hughes and R.L. Taylor, The linear Triangular plate bending element, In J.R. Whiteman, editor, The Mathematics of Finite Elements and Applications IV, MAFELAP, 1981, pp. 127-142, Academic Press, 1982.

[35] F. Kikuchi and K. Ishii, An improved 4-node quadrilateral plate bending element of the Reissner-Mindlin type, Comput. Mech., 23 (1999), pp. 240-249.

[36] E. Liberman, A posteriori error estimator for a mixed finite element method for ReissnerMindlin plate, Math. Comp., 70 (2001), pp. 1383-1396. MR1836909 (2003c:74094)

[37] C. Lovadina and R. Stenberg, A posteriori error analysis of the linked interpolation technique for plate bending problems, SIAM J. Numer. Anal., 43 (2005), 2227-2249. MR2192338 (2006i:65198)

[38] M. Lyly, On the connection between some linear triangular Reissner-Mindlin plate bending elements, Numer. Math., 85 (2000), pp. 77-107. MR1751364 (2001b:65127)

[39] P.B. Ming and Z.C. Shi, Quadrilateral mesh, Chinese Annals of Mathematics, 23B (2002), pp. 1-18.

[40] J. Pitkåranta and M. Süri, Design principles and error analysis for reduced-shear plate bending finite elements, Numer. Math., 75 (1996), pp. 223-266. MR1421988 (98c:73078) 
[41] L. R. Scott, S. Zhang, Finite element interpolation of nonsmooth functions satisfying boundary conditions, Math. Comp., 54 (1990), pp. 483-493. MR1011446 (90j:65021)

[42] Z.C. Shi, A convergence condition for quadrilateral Wilson element, Numer. Math. 44 (1984), pp. 349-361. MR757491 (86d:65151)

[43] R. Stenberg and M. Süri, An hp error analysis of MITC plate elements, SIAM. J. Numer. Anal., 34 (1997), pp. 544-568. MR1442928 (98g:65112)

[44] M. Süri and I. Babuška, C. Schwab, Locking effects in the finite element approximation of plate models, Math. Comp., 64 (1995), pp. 461-482. MR.1277772 (95f:65207)

[45] A. Tessler and T. J. R. Hughes, An improved treatment of transverse shear in the Mindlin type four-node quadrilateral element, Comp. Meths. Appl. Mech. Engrg., 39 (1983), pp. 311-335.

[46] P.A.Raviart and J.M.Thomas, A mixed finite element method for second order elliptic problems, Proc. Sympos. Mathematical Aspects of the Finite Element Method (Rome, 1975), Lecture Notes in Math., 606 (1977), pp. 292-315, Springer-Verlag. MR0483555 (58:3547)

[47] R. Verfürth, A Review of A Posteriori Error Estimation and Adaptive Mesh-Refinement Techniques, Wiley-Teubner, 1996.

[48] O.C. Zienkiewicz, R.L. Taylor and J.M. Too, Reduced integration Technique in general analysis of plates and shells, Int. J. Numer. Meth. Engrg., 3 (1971), pp. 275-290.

Institut für Mathematik, Humboldt Universität zu Berlin, Unter den Linden 6, D-10099 Berlin, Germany

E-mail address: cc@math.hu-berlin.de

Lmam and School of Mathematical Sciences, Peking University, Beijing 100871, People's Republic of China

E-mail address: hujun@math.pku.edu.cn 